Helgoländer wiss. Meeresunters. 32, 476-507 (1979)

\title{
Quantitative ökologische Analyse eines Rifflagunenareals bei Aqaba (Golf von Aqaba, Rotes Meer)*
}

\author{
H. Mergner \\ Ruhr-Universität Bochum, Lebrstubl für Spezielle Zoologie; \\ D-4630 Bochum, Bundesrepublik Deutschland
}

\begin{abstract}
Quantitative ecological analysis of a reef lagoon area near Aqaba (Gulf of Aqaba, Red Sea). Following previous investigations on a fringing reef in the Red Sea near Aqaba (Mergner \& Schuhmacher, 1974; Mergner \& Svoboda, 1977) this paper presents a quantitative analysis of the faunal and floral stock in one of the selected test areas $(5 \times 5 \mathrm{~m})$ located in the reef lagoon studied. During the observation period 237 macrobenthic species of 192 genera were found. Species numbers were 22 (algae), 18 (cnidarians), 22 (crustaceans), 113 (molluscs), 27 (echinoderms), 14 (fishes) and 21 (smaller animal taxa including foraminiferans, sponges, turbellarians, polychetes and ascidians). These numbers, though derived from a small and relatively monotonous reef area, exceed by far all data known from comparable reef zones. The ecological conditions as well as habitat preferences, times of occurrence, seasonal abundances, species diversities and dominances of the inhabitants observed in the test area are discussed. Comparisons of the population data of October, 1975 and March, 1976 indicate the influence of the succession, abundance and disappearance of the phytal on the environmental conditions of the mobile fauna, for example molluscs, echinoderms and fishes. The increase of some of its species, especially of juvenile individuals, is remarkable. Within the same period juvenile colonies of some xeniids and the stony coral Stylophora pistillata undergo similar seasonal fluctuations; colonies eliminated by grazers or other, unknown circumstances, are replaced by new settlers. Altogether, the faunal and algal stock of this lagoon area can be interpreted as a climax community; its impressive density is apparently the result of a high number of various microhabitats. Each of these ecological niches provides manyfold living spaces for specific communities with different adaptive strategies. The highest number of species plus the highest diversity indices rank mostly among the mobile groups.
\end{abstract}

\section{EINLEITUNG}

Die erste subtile ökologische Analyse eines Saumriffes wurde von Mergner \& Schuhmacher (1974) an zwei Riffabschnitten südlich von Aqaba (Rotes Meer) durchgeführt. Dabei wurden vor allem die biophysiographischen Riffzonen festgelegt, die ihnen zugehörigen Lebensgemeinschaften beschrieben und ihre Abhängigkeit von Wasseraustausch, Beleuchtung und Sedimentationsrate verdeutlicht. Diese Untersuchungen wurden von Mergner \& Svoboda (1977) zu funktionsökologischen Analysen der jahreszeitlichen Veränderungen des Makrobenthos innerhalb ausgewählter Testquadrate erweitert und

\footnotetext{
* Mit Unterstützung der Deutschen Forschungsgemeinschaft.
} 
durch produktionsbiologische Messungen ergänzt (Svoboda, 1978). Weitere faunistische und synökologische Probleme wurden im gleichen Riffgebiet von Mergner \& Mastaller (1979, im Druck) am Lagunen-Testareal L-1, von Mergner \& Schuhmacher (in Vorbereitung) am Vorriff-Testareal U-7 sowie von Mastaller an Mollusken und Echinodermen und von Nobbe an Poriferen durchgeführt (beide in Vorbereitung).

Bei der Veröffentlichung der vorläufigen Untersuchungsergebnisse (1977) wurden jedoch - mit Ausnahme der benthischen Makroalgen - noch keine exakten quantitativen Daten zum Artenbestand gegeben und eine genaue Analyse der Habitatpräferenz und saisonalen Abundanz seiner Einzelarten ausgespart. Sie sollen nunmehr für alle $237 \mathrm{im}$ Testareal L-1 festgestellten Makrobenthos-Arten vorgelegt und durch Zahlenmaterial, Diversitätsberechnungen und Verteilungspläne aller wesentlichen Benthos-Gruppen sowie eine synökologische Zusammenschau vervollständigt werden.

\section{METHODIK}

Uber die prinzipiellen Naturverhältnisse des Golfes von Aqaba haben Mergner \& Schuhmacher (1974) unter Einbeziehung früherer Erkenntnisse ausführlich berichtet. Ihre Angaben sollen daher nur für den Untersuchungszeitraum im Winterhalbjahr 1975/76 und den Bereich des Testareals L-1 in der Rifflagune ergänzt werden: So übertrafen die mittleren Tagestemperaturen des Dezembers die langjährigen Mittelwerte, lagen aber im Februar und März unter diesen. Der Wind kam fast durchwegs aus nördlichen Richtungen, in der ersten Hälfte des Untersuchungszeitraumes häufiger aus NO bis NNO, in der zweiten dagegen häufiger aus NNO bis N. Insgesamt überwogen mittlere Windgeschwindigkeiten von 2-4 Bft. mit 65-91\% Häufigkeit, in der ersten Hälfte sogar mit 86-91\% gegenüber $65-73 \%$ in der zweiten, in der wiederum sowohl windstille Perioden wie auch höhere Windgeschwindigkeiten von 5-6 Bft. deutlich zunahmen.

Der normale Tidenhub beträgt im nördlichen Golf von Aqaba $0,4-0,7 \mathrm{~m}$, bei Springtiden bis 1,2 m. Extremes NW mit 1,1 m herrschte u. a. am 16./17. Februar 1976. Zur gleichen Zeit wurden von der Marine Science Station Aqaba an der Wasseroberfläche ein Salzgehalt von 40,8\%o und ein pH-Wert von 8,4 gemessen sowie in $10 \mathrm{~m}$ Tiefe 40,6\%o und 8,3. Damit stimmen die feldmäßig gewonnenen Werte (Aerometer: 41 \%; Merck-pHPapier: 8,3) gut überein. Der $\mathrm{O}_{2}$-Gehalt betrug bei $19,9^{\circ} \mathrm{C}$ Oberflächentemperatur $7,8 \mathrm{mg}$ $\mathrm{O}_{2} / 1$. Die mittleren Monatstemperaturen des Oberflächenwassers schwankten zwischen 24,5 ${ }^{\circ} \mathrm{C}$ im Oktober 1975 und 21,1 ${ }^{\circ} \mathrm{C}$ im Februar 1976. Über die Meßwerte zur Oberflächen- und bodennahen Strömung wird im Kapitel "Untersuchungsgebiet" berichtet.

Um die Ausgangssituation im Testareal L-1 für die nachfolgenden Untersuchungen über saisonale Abundanzänderungen während des Winterhalbjahres 1975/76 nicht unwiederbringlich zu verfälschen, wurden im Oktober 1975 nur sessile Tiergruppen und die meisten Echinodermen-Arten quantitativ erfaßt. Von den übrigen Gruppen wurden dagegen unter größter Schonung des Biotopes nur Belegproben entnommen. Im Frühjahr 1976 wurde eine vollständige Bestandsaufnahme aller Makrobenthos-Arten durchgeführt und durch M. Mastaller für Mollusken und Echinodermen noch mehrfach bis zum Herbst 1977 wiederholt. Hierzu wurde das teilweise sehr dichte Phytal gründlich durchsucht. Alle 
lose liegenden Hartsubstratbrocken wurden umgedreht, sofern erforderlich, im Labor zerkleinert und anschließend in Süßwasser unter Formolzusatz eingelegt, um durch Verschlechterung der Lebensbedingungen die kryptische Fauna aus ihren Schlupfwinkeln zu treiben. Die Fauna der Sandmulden wurde quantitativ bis $15 \mathrm{~cm}$ Tiefe mit Hilfe eines genormten Metallstechkastens gewonnen, aus dessen offener Seite der Inhalt durch entsprechend weitmaschige Siebe geschaufelt wurde. Auf Schwierigkeiten stieß die quantitative Analyse nur bei einigen streng kryptisch lebenden Arten wie bei der Foraminifere Homotrema rubrum, der Spirorbide Pileolaria militaris und vielen Ophiuriden. Sie finden sich meist in schwer zugänglichen Bodenspalten oder unter größeren Korallenfelsstrukturen und sind daher ohne Zerstörung ihrer Verstecke kaum vollständig zu erlangen. Bei allen anderen sessilen und mobilen Makrobenthos-Gruppen können die Angaben zur Abundanz und relativen Häufigkeit als nahezu komplett gelten und eindeutige Aussagen zur Populationsdynamik und Artendiversität gemacht werden. Daneben wurden auch die jeweiligen Habitatpräferenzen und Ernährungsweisen festgestellt. Mastaller (in Vorbereitung) wird noch ausführlich über trophische Beziehungen bei Mollusken und Echinodermen auf Grund der Ergebnisse von Mageninhaltsanalysen berichten.

Zur genauen Lokalisierung der Einzelfundplätze und Flächenberechnung der kolonial wachsenden Arten wurde das Testareal mit einem $5 \times 5-\mathrm{m}-\mathrm{Netz}$ aus Perlonleinen von $1 \mathrm{~m}$ Maschenweite überspannt und auf den jeweils bearbeiteten Quadratmeter ein gleichgroßer Testrahmen mit einem Netz von $10 \mathrm{~cm}$ Maschenweite aufgelegt. Mit seiner Hilfe konnten alle Arten - die oben genannte Kryptofauna, kleinste feinfädige Algenrasen und natürlich alle Fische ausgenommen - in ihrer derzeitigen Position bei senkrechter Projektion lagerichtig und, soweit erforderlich, flächentreu kartiert werden. $\mathrm{Da}$ zahlreiche Arten vor allem der mobilen Fauna überwiegend verborgen leben, ist ihr Versteck meist unter den im Lageplan am jeweiligen Standort dargestellten Strukturen zu suchen. Durch wiederholtes Kartieren und Auswerten aller zeichnerischen und photographischen Protokolle ergab sich schließlich ein fast lückenloses Bild von der Besiedlungsstruktur des Testareals, von der Zusammensetzung seiner Lebensgemeinschaften und von den während des Untersuchungszeitraumes erfolgten Abundanzänderungen.

Mastaller (in Vorbereitung) hat bei seinen Abundanzuntersuchungen an Mollusken und Echinodermen im gleíchen Riffgebiet der Vergrößerung des dreidimensional verfügbaren Siedlungsraumes gegenüber der in die Horizontale projizierten Flächeneinheit durch Berücksichtigung des Oberflächenindex OI Rechnung getragen. Bei der Bearbeitung des Testareals L-1 erschien dies jedoch wegen der geringen Anzahl und Ausmaße der hier vorhandenen Strukturaufbauten und ihrer unterschiedlichen Signifikanz für die Bewohner nicht erforderlich. Zur Berechnung der Artendiversität und Dominanz (zahlenmäßige Abundanz) der einzelnen Benthosgruppen durch M. Mastaller wurde der "Shannon Weaver" - Index (vgl. Pielou, 1969) verwendet (Tab. 9):

$$
H=-\sum_{i=1}^{N} p_{i} \ln p_{i}
$$

Dabei bedeuten $\mathrm{N}$ die Artenzahl in jeder Gruppe und $\mathrm{p}_{\mathrm{i}}$ die gemessene Individuenrate. (Weitere ökologisch orientierte Erläuterungen finden sich bei Mastaller, in Vorbereitung.) 
Tabelle 1

Von Oktober 1975 bis November 1977 im Testareal L-1 festgestellte Arten des Makrobenthos (Anzahl der Gattungen und Arten in Klammern; insgesamt 192 Gattungen, 237 Arten)

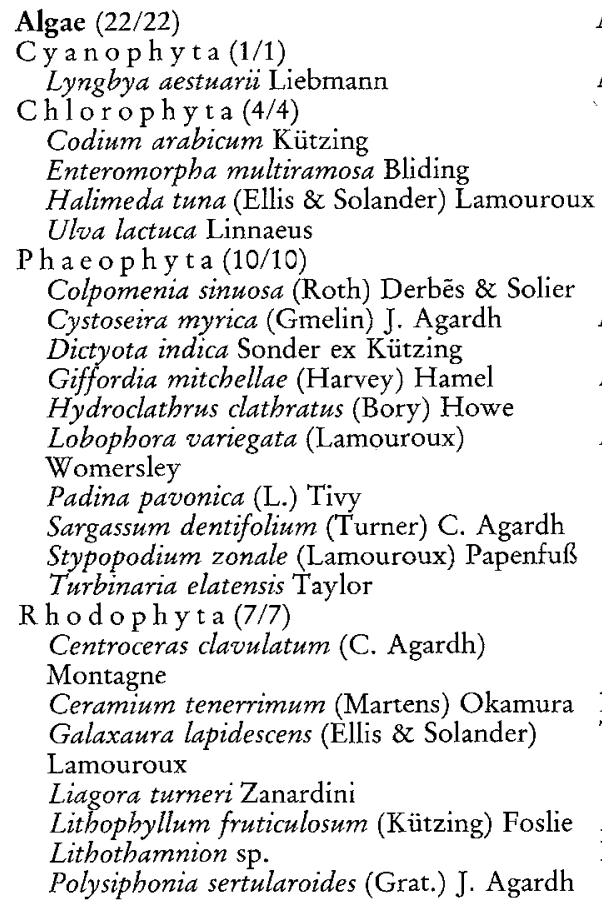

Protozoa (2/2)

For a min if e r a $(2 / 2)$

Homotrema of. rubrum (Lamarck)

Maginopora sp.

Porifera (11/11)

Calcarea $(1 / 1)$

Leucosolenia tenuipilosa Dendy

Demospongia e $(10 / 10)$

Adocia dendyi (Burton)

Biemna mucronata Koltun

Chondrillastra mixta (Schulze)

Cliona vastifica (Hancock)

Dysidea cinerea Keller

Erylus proximus Dendy

Fasuberea quadrangulata Lévi

Rhaphoxya typica Hallmann

Spongia officinalis Linné, var. arabica Keller Tethya seychellensis (Wright)

Cnidaria $(16 / 18)$

Hydrozoa, Hydroidea (1/1) Millepora exaesa Forskål
Anthozoa, Ceriantharia (1/1) Cerianthus sp.

Anthozoa, Scleractinia (7/7) Cyphastrea microphthalma (Lamarck) Favia pallida (Dana)

Favites melicerum (Esper)

Goniastrea retiformis (Lamarck)

Montipora tuberculosa (Lamarck)

Pavona decussata (Dana) Stylophora pistillata (Esper)

Anthozoa, Zoantharia (1/1) Palythoa tuberculosa Esper

Anthozoa, Stolonifera (1/1) Tubipora musica Linnaeus

Anthozoa, Alcyonaria (5/7) Cladiella pachyclados (Klunzinger) Litbopbyton arboreum Forskål Parerythropodium fuloum (Forskål) Sinularia leptoclados (Ehrenberg) Sinularia polydactyla (Ehrenberg) Xenia macrospiculata Gohar Xenia membranacea Schenk

Plathelminthes $(1 / 1)$

Turbellaria, Polycladida (1/1) Stylochidae

Annelida (6/6)

Polychaeta, Errantia (3/3) Ipbione muricata (Savigny) Nereis sp. Subadyte pellucida (Ehlers)

Poly chaet a, Sedentaria (3/3) Pileolaria militaris Claparede Sabellastarte indica Savigny Spirobranchus giganteus corniculatus (Grube)

Arthropoda, Crustacea (19/22)

S tom a to pod a (1/2) Gonodactylus chiragra (Fabricius) Gonodactylus falcatus (Forskål)

Decapoda, Natania (3/5) Alpheus insignis Heller Alpheus sp. Athanas djiboutensis Coutière Athanas sp. Thor amboinensis

Decapoda, Anomura (5/5) Calcinus latens ( $R$ andall) Clibanarius signatus Heller Galathea affinis Ortmann Petrolisthes leptocbeles (Heller) Pisidia inaequalis (Heller) 
Tabelle 1 (1. Fortsetzung)

Decapoda, Brachyura (8/8) Elamena mathaei (Desmarest)

Lybia heterochelis (Zehntner)

Medaeus neglectus Balss

Menaethiops sp.

Menaethius monoceros (Latreille)

Micippa platypes Rüppell

Portunus alcocki (Nobili)

Trapezia cymodoce (Herbst)

I s o p o d a (1/1)

Cymodoce sp.

A mphipoda (1/1)

Gammaridae

Mollusca (77/113)

Amphine ura (2/3)

Ischnocbiton yerburi Smith

Tonicia costata Leloup

Tonicia suezensis (Reeve)

$\mathrm{G}$ as tropoda (50/83)

Ancilla lineolata Adams

Apollon pusillus Broderip

Balcis cf. acuta Adams

Cerithium caeruleum Sowerby

Cerithium columna Sowerby

Cerithium nesioticum Pilsbry \& Vanatta

Cerithium nodulosum erythraeonense

Lamarck

Cerithium rüeppelli Philippi

Cerithium scabridum Philippi

Clypeomorus tuberculatus (L.)

Conus arenatus Hwass in Brugière

Conus coronatus Gmelin

Conus lividus Hwass in Brugière

Conus spirigloxus Deshayes

Conus striatus $\mathrm{L}$.

Conus taeniatus Hwass in Brugière

Conus tessulatus Born

Conus textile $\mathrm{L}$.

Coralliophilia violacea (Kiener)

Cymatium pileare (L.)

Cymatium rubecula (L.)

Cypraea arabica $\mathrm{L}$.

Dendropoma maximum (Sowerby)

Drupa digitata Lamarck

Drupella rugosa (Born)

Fusinus polygonoides (Lamarck)

Gena auricularia Lamarck

Gena varia A. Adams

Gibbula declivis (Forskål)

Haliotis pustulata Reeve

Harpa minor Lamarck

Heliacus variegatus (Gmelin)

Hemitoma tricarinata (Born)

Hydatina physis Lamarck

Lambis truncata sebae (Kiener)

Latirus turritus (Gmelin)
Maculotriton cf. serriale Deshayes

Marginella savignyi Issel

Melampus flavus (Gmelin)

Mitra cucumerina Lamarck

Mitra fasciolaris Deshayes

Mitra litterata Lamarck

Mitra rufomaculata Sowerby

Mitrella sp.

Morula granulata (Duclos)

Morula uva (Röding)

Nassa serta (Brugière)

Nassarius albescens gemmuliferus (Adams)

Nassarius arcularia plicatus (Röding)

Neritopsis radula $(\mathbf{L}$.)

Planaxis lineolatus $\mathrm{Da}$ Costa

Pleurotoma ssp.

Polinices mammilla (L.)

Polinices melanostoma (Gmelin)

Rbinoclavis aspera (L.)

Royella sinon (Bayle)

Scala jomardi (Audouin)

Scala lamellosa (Lamarck)

Serpulorbis inopertus (Rüppell)

Stomatia phymotis Helbling

Strombus erytbrinus Dillwyn

Strombus fusiformis Sowerby

Strombus gibberulus albus Mörch

Strombus mutabilis ocbroglottis Abbott

Strombus tricornis Humphrey

Subancilla annulata (Reeve)

Subancilla flammea (Quoy \& Gaimard)

Terebra affinis Gray

Terebra babylonia Lamarck

Terebra crenulata (L.)

Terebra flavofasciata Pilsbry

Thais tuberosa (Röding)

Trivia oryza (Lamarck)

Trocbus dentatus Forskal

Trochus sp.

Turbo radiatus (Gmelin)

Turitella maculata (Reeve)

Vexillum amabile (Reeve)

Vexillum infaustum (Reeve)

Vexillum leucozonias Deshayes in Labarde

Vexillum pardalis (Kuster)

Volema pyrum nodosum (Lamarck)

Xenoturris cingulifera Weinkauff

$\mathrm{Scaphopoda}(1 / 2)$

Dentalium elephantinum $\mathbf{L}$.

Dentalium cf. octangulum Donovan

Bivalvia (24/25)

Arca antiquata $\mathrm{L}$.

Arca plicata Chemnitz

Asaphis deflorata (L.)

Barbatia lacerata (L.)

Cardium anstrale Sowerby

Cardita variegata Brugière 
Tabelle 1 (2. Fortsetzung)

Chama sp.

Codackia divergens (Philippi)

Cyclotellina scobinata (L.)

Gastrochaena sp.

Glycymeris pedunculus (L.)

Hemicardium fragum (L.)

Isognomon sp.

Lima lima (L.)

Loripes erythraeus (Issel)

Lucina edentula (L.)

Modiolus auriculatus (Krauss)

Notirus macrophyllus (Deshayes)

Pecten sp.

Pedum spondyloideum (Gmelin)

Pinctada radiata (Leach)

Pinna bicolor Gmelin

Streptopinna saccata (L.)

Tapes litteratus (L.)

Tridacna squamosa Lamarck

Echinodermata (23/27)

Holothuroidea (3/4)

Halodeima atra (Jäger)

Halodeima edulis (Lesson)

Holotburia pardalis (Selenka)

Pseudocnus nov. spec.

Echinoidea (10/10)

Clypeaster bumilis (Leske)

Diadema setosum (Leske)

Echinometra mathaei (Blainville)

Eucidaris metularia (Lamarck)

Heterocentrotus mammillatus (L.)

Lovenia elongata (Gray)

Metalia spatagus (L.)

Microcyphus rousseaui Agassiz

Paraster gibberulus (Agassiz)

Tripneustes gratilla (L.)

A s t e roide a (3/3)

Asterina burtoni Gray
Astropecten hemprichi Müller \& Troschel

Linckia multiflora (Lamarck)

Ophiuroidea (7/10)

Amphipholis squamata (Delle Chiaje)

Dougaloplus echinatus (Ljungmann)

Ophiocoma erinaceus Müller \& Troschel

Ophiocoma pica Müller \& Troschel

Ophiocoma scolopendrina (Lamarck)

Ophiolepis cincta Müller \& Troschel

Ophionereis dubia Müller \& Troschel

Ophionereis porrecta Lyman

Ophiothrix propinqua Lyman

Ophiura kinbergi (Ljungmann)

Tunicata (1/1)

Ascidiacea, Pleurogona (1/1)

Didemnum moseleyi Herdmann

Vertebrata, Pisces (14/14)

My ctophiformes (1/1)

Synodus variegatus (Lacépède)

Anguillif ormes (1/1) Siderea grisea (Lacépède)

Pleuronectiformes $(\mathbf{1 / 1})$

Botbus pantherinus Rüppell

Perciformes $(10 / 10)$

Amphiprion bicinctus Rüppell

Chaetodon chrysurus paucifasciatus Ahl

Dascyllus trimaculatus Rüppell

Dendrochirus brachypterus

(Cuvier \& Valenciennes)

Meiacanthus nigrolineatus Smith-Vaniz

Paracirrbites forsteri (Bloch \& Schneider)

Platycephalus indicus (Linnaeus)

Pterois volitans (Linnaeus)

Pteropterus radiatus (Cuvier \& Valenciennes)

Synanceichthys verrucosa (Bloch \& Schneider)

Tetrad ontif ormes (1/1)

Hemibalistes chrysopterus (Bloch \& Schneider)

\section{ERGEBNISSE}

\section{Untersuchungsgebiet}

Tabelle 1 gibt eine Zusammenstellung aller im Testareal L-1 während der Untersuchungsperiode festgestellten systematischen Kategorien des Makrobenthos mit der jeweiligen Anzahl ihrer Gattungen und Arten. Dieses Areal liegt mit einer Fläche von $5 \times 5 \mathrm{~m}$ (25 $\mathrm{m}^{2}$ ) im südlichen Teil der Rifflagune, $1 \mathrm{~km}$ südlich der neuerbauten Marine Science Station Aqaba und 56,5-61,5 m von der mittleren Hochwasserlinie entfernt (vgl. Fig. 1 in: Mergner \& Svoboda, 1977). Bei ihrer biophysiographischen Zonierung des Lagunensaumriffes haben Mergner \& Schuhmacher (1974) diesen Bereich der Sargassum dentifolium-Zone, 
nahe der Grenze zur Stypopodium zonale-Zone, zugeteilt. Diese Entscheidung wurde beim Aufkommen des Algenwuchses im Frühjahr 1976 durch das Uberwiegen beider Algenarten mit 18,9 bzw. 2,2 \% Gesamtflächenbedeckung und 72,5 bzw. 8,3\% des Algenbestandes eindeutig bestätigt (Tab. 2). Andererseits weist das Vorkommen üppiger Weichkorallenbestände auf einem breiten, fast algenfreien Arealstreifen auf die enge Verzahnung der Braunalgenzone mit der seewärts anschließenden Sinularia-Cladiella-Zone hin. Außerdem zeigen stark erodierte Korallenfelshorste, kugelige Kolonien massig wachsender Steinkorallen und einige Mikroatolle die Zugehörigkeit des Testareals zur übergeordneten Horstzone als dem charakteristischen Mittelabschnitt der Rifflagune.

Die Strukturanalyse des Testareals L-1 vom Oktober 1975 (Abb. 1) gibt eine einförmige Unterwasserlandschaft wieder: Der Lagunenboden besteht aus nach Süden hin leicht geneigten Korallenfelsplatten, die als Überreste der einstigen, durch Wassererosion zerstörten Riffplattform anzusehen sind. Ihre tiefer liegenden Mulden bedecken feiner Sandmulm vorwiegend organogener Herkunft und einzelne Areale zusammengeschwemmter Korallenbruchstücke und Molluskenschalen. Vom Lagunenboden erheben sich nur wenige größere Aufbauten wie die beiden Mikroatolle mit je $75 \mathrm{~cm}$ Durchmesser, massig, krustenförmig oder verzweigt wachsende lebende Steinkorallenkolonien und eine Anzahl toter Korallenfelsruinen. Offensichtlich bieten aber ihre Höhlungen, Löcher und Spalten sowie die Unterseiten erodierter Bodenplatten als Hartsubstratstrukturen zusammen mit den Weichkorallenbeständen einer großen Arten- und Individuenzahl ausreichenden Lebensraum. Dafür spricht die auffallend dichte Besiedlung mit Vertretern der Makrofauna schon vor Aufkommen des Algenwuchses zu Jahresbeginn.

Begünstigt wird diese Situation neben der guten Durchleuchtung der obersten Wasserschichten vor allem durch einen optimalen Wasseraustausch, der - ohne zerstörende Brandungskräfte und nur selten bei schwerem Seegang - fast ausschließlich über die Rifflängsströmung erfolgt: Unabhängig vom Gezeitenstand verlief die Oberflächenströmung im Bereich des Testareals L-1 während des. Winterhalbjahres 1975/76 fast genau nordsüdwärts (Abb. 2). Dabei wurden im Februar 1976 bei einer Windstärke von $5 \mathrm{Bft}$. und Nordwind in $1 \mathrm{~m}$ Höhe über dem Lagunenboden Strömungsgeschwindigkeiten von $10,1-10,8 \mathrm{~cm} / \mathrm{sec}$ gemessen. Zum Feststellen der Abdrift wurden an mehreren Tagen des Februars insgesamt 176 Fluorescinproben eingebracht. Mit ihrer Hilfe konnte die Abhängigkeit der bodennahen Strömung in 10-15 cm Höhe über dem Lagunenboden vom jeweiligen Gezeitenstand eindeutig nachgewiesen werden: Bei auflaufendem Wasser (1 Std. nach Niedrigwasser) zeigte die Bodenströmung gegenüber der Oberflächenströmung Abweichungen von $30-35^{\circ}$ nach SO, bei ablaufendem Wasser (1 Std. vor NW) solche von $30-45^{\circ}$ nach SW (Abb. 2). Es entspricht den Ergebnissen langjähriger Beobachtungen zum Korallenwachstum, daß die strömungszugewandten Seiten der emporragenden Mikroatolle den weit überwiegenden Anteil lebender Korallenwuchsflächen zeigten.

\section{Saisonale Bestandsänderungen in L-1}

Wegen des Ausbleibens schwerer Südstürme im Golf von Aqaba während des Winterhalbjahres 1975/76 ergaben sich keine wesentlichen Strukturveränderungen im Bereich des Testareals L-1 oder nachteilige Folgen für die dort angesiedelte Makrofauna. 


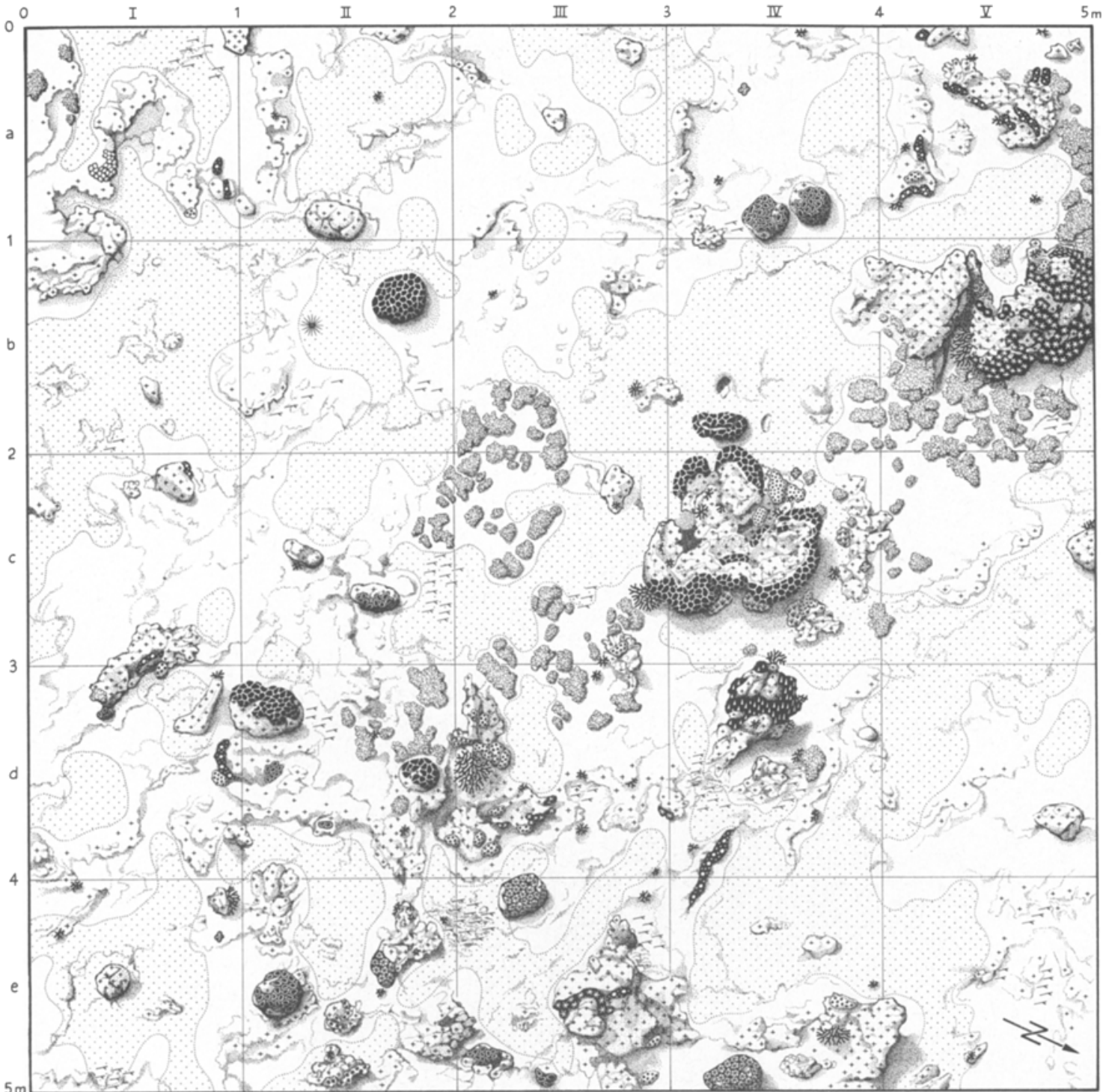

Testquadrat $L-1$, Strukturanalyse

Ausgangssituation Oktober 1975

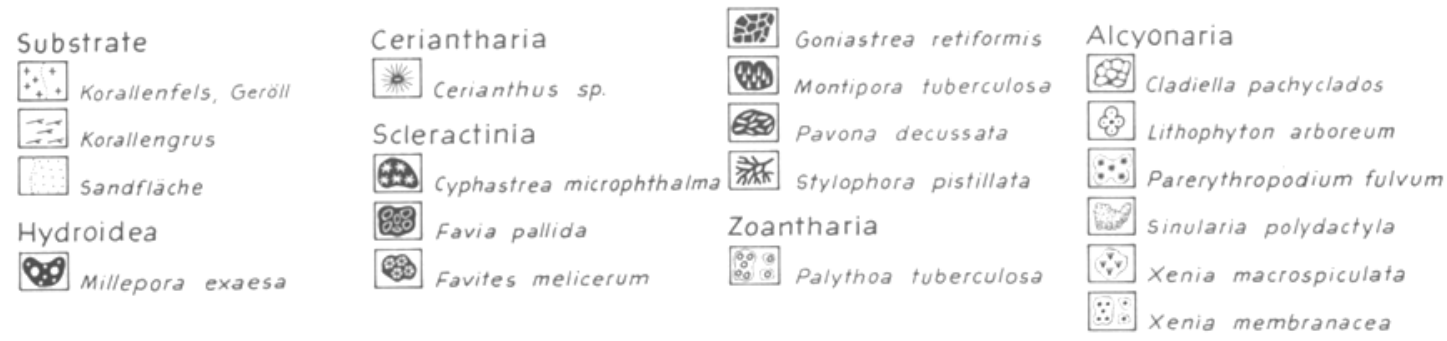

Abb. 1: Ausgangssituation im Testareal L-1 im Oktober 1975 mit Analyse der Substratstrukturen und Korallenbesiedlung 
Lediglich kleinere Bruchschäden an Stylophora pistillata-Kolonien und die Verlagerung einiger losgerissener Weichkorallenbüschel wurden registriert. Dagegen erfuhren die Makrobenthos-Gemeinschaften teilweise bemerkenswerte saisonale Veränderungen, die im Folgenden an Hand von Lageplänen und Tabellen kommentiert werden sollen. Da aus bereits erwähnten Gründen im Oktober 1975 nur sessile Tiergruppen quantitativ erfaßt werden konnten, gewinnt die herbstliche Bestandsaufnahme der Echinodermen als einziger mobilen Gruppe Modellcharakter für den Vergleich mit dem Frühjahrsaspekt nach Aufkommen des Algenwuchses.

\section{Algenbedeckung}

Die auffälligsten Veränderungen im Testareal L-1 zwischen Herbst 1975 und Frühjahr 1976 betrafen das Phytal der Rifflagune. Bis Ende Oktober 1975 war der Algenwuchs völlig verschwunden (Abb. 1). Lediglich kurze Stümpfe der Algenbüschel haben den

Tabelle 2

Habitatpräferenz, saisonale Abundanz und Flächenanteile der benthischen Algen im Testareal L-1 (Frühjahr 1976). Mit " bezeichnete Arten sind in Abb. 3 bei senkrechter Projektion lagerichtig und flächentreu eingetragen. *** phy - Phytal, Bereich geschlossenen Algenwuchses; sm - Sandmulmbedeckte Flächen mit Korallenschutteinlagerungen; mi - Mikroatolle und aufragende tote Korallenfelshorste; crs - Lose liegende Korallenfelsplatten

\begin{tabular}{|c|c|c|c|c|c|}
\hline Species & $\begin{array}{l}\text { Bevorzugtes } \\
\text { Habitat bzw. } \\
\text { Substrat*: }\end{array}$ & $\begin{array}{l}\text { Saisonale Ab } \\
\text { Monate der } \\
\text { Optimalent- } \\
\text { wicklung }\end{array}$ & $\begin{array}{l}\text { undanz im } F \\
\text { Anzahl der } \\
\text { Kolonien } \\
\text { bzw. Thalli }\end{array}$ & $\begin{array}{l}\text { Anteil in \% } \\
\text { der Gesamt- } \\
\text { arealfläche }\end{array}$ & $\begin{array}{l}\text { bezogen auf: } \\
\text { Anteil in \% } \\
\text { der Gesamt- } \\
\text { algenfläche }\end{array}$ \\
\hline * Lyngbya aestuarii & $\mathrm{sm}, \mathrm{crs}$ & I-IV & 4 & 0,01 & 0,05 \\
\hline $\begin{array}{l}\text { Codium arabicum } \\
\text { Enteromorpha }\end{array}$ & phy, sm, crs & $\mathrm{I}-\mathrm{IV}$ & 4 & 0,04 & 0,18 \\
\hline multiramosa & $\mathrm{mi}, \mathrm{crs}$ & ganzjährig & - & 0,01 & 0,02 \\
\hline * Halimeda tuna & $\mathrm{mi}$, crs & $\mathrm{I}-\mathrm{IV}$ & 97 & 0,69 & 2,61 \\
\hline$*$ Ulva lactuca & $\mathrm{sm}, \mathrm{crs}$ & I-III & 1 & 0,01 & 0,02 \\
\hline * Colpomenia sinuosa & $\mathrm{sm}, \mathrm{crs}$ & I-III & 7 & 0,06 & 0,25 \\
\hline * Cystoseira myrica & phy, sm, crs & XII-III & 25 & 0,43 & 1,61 \\
\hline * Dictyota indica & phy, sm, crs & II-IV & 142 & 1,92 & 7,36 \\
\hline Giffordia mitchellae & mi, crs & ganzjährig & - & 0,01 & 0,01 \\
\hline * Hydroclatbrus clatbratus & $\mathrm{sm}, \mathrm{crs}$ & II-IV & 2 & 0,15 & 0,58 \\
\hline * Lobophora variegata & $\mathrm{mi}, \mathrm{crs}$ & ganzjährig & 66 & 0,51 & 2,16 \\
\hline - Padina pavonica & $\mathrm{sm}, \mathrm{mi}, \mathrm{crs}$ & XI-III & 7 & 0,09 & 0,34 \\
\hline * Sargassum dentifolium & $\mathrm{sm}, \mathrm{crs}$ & XII-IV & 1470 & 18,89 & 72,45 \\
\hline * Stypopodium zonale & $\mathrm{mi}, \mathrm{crs}$ & II-IV & 176 & 2,18 & 8,33 \\
\hline Turbinaria elatensis & $\mathrm{mi}, \mathrm{crs}$ & IV-VIII & - & - & - \\
\hline Centroceras clavulatus & $\mathrm{mi}, \mathrm{crs}$ & ganzjährig & - & 0,01 & 0,01 \\
\hline Ceramium tenerrimum & $\mathrm{mi}$, crs & ganzjährig & - & 0,01 & 0,01 \\
\hline * Galaxaura lapidescens & crs & II-IV & 1 & 0,09 & 0,35 \\
\hline * Liagora turneri & crs & II-IV & 5 & 0,67 & 2,56 \\
\hline * Lithopbyllum & & & & & \\
\hline fruticulosum & $\mathrm{mi}, \mathrm{crs}$ & ganzjährig & 3 & 0,05 & 0,21 \\
\hline $\begin{array}{l}\text { Litbothamnion sp. } \\
\text { Polysiphonia }\end{array}$ & $\mathrm{mi}, \mathrm{crs}$ & ganzjährig & 27 & 0,23 & 0,88 \\
\hline sertularoides & $\mathrm{mi}, \mathrm{crs}$ & ganzjährig & - & 0,01 & 0,01 \\
\hline Gesamtkolonieanzahl und $\mathrm{F}$ & ichenanteile & & 2037 & 26,07 & 100,00 \\
\hline Algenfreies Areal & & & - & 73,93 & - \\
\hline
\end{tabular}




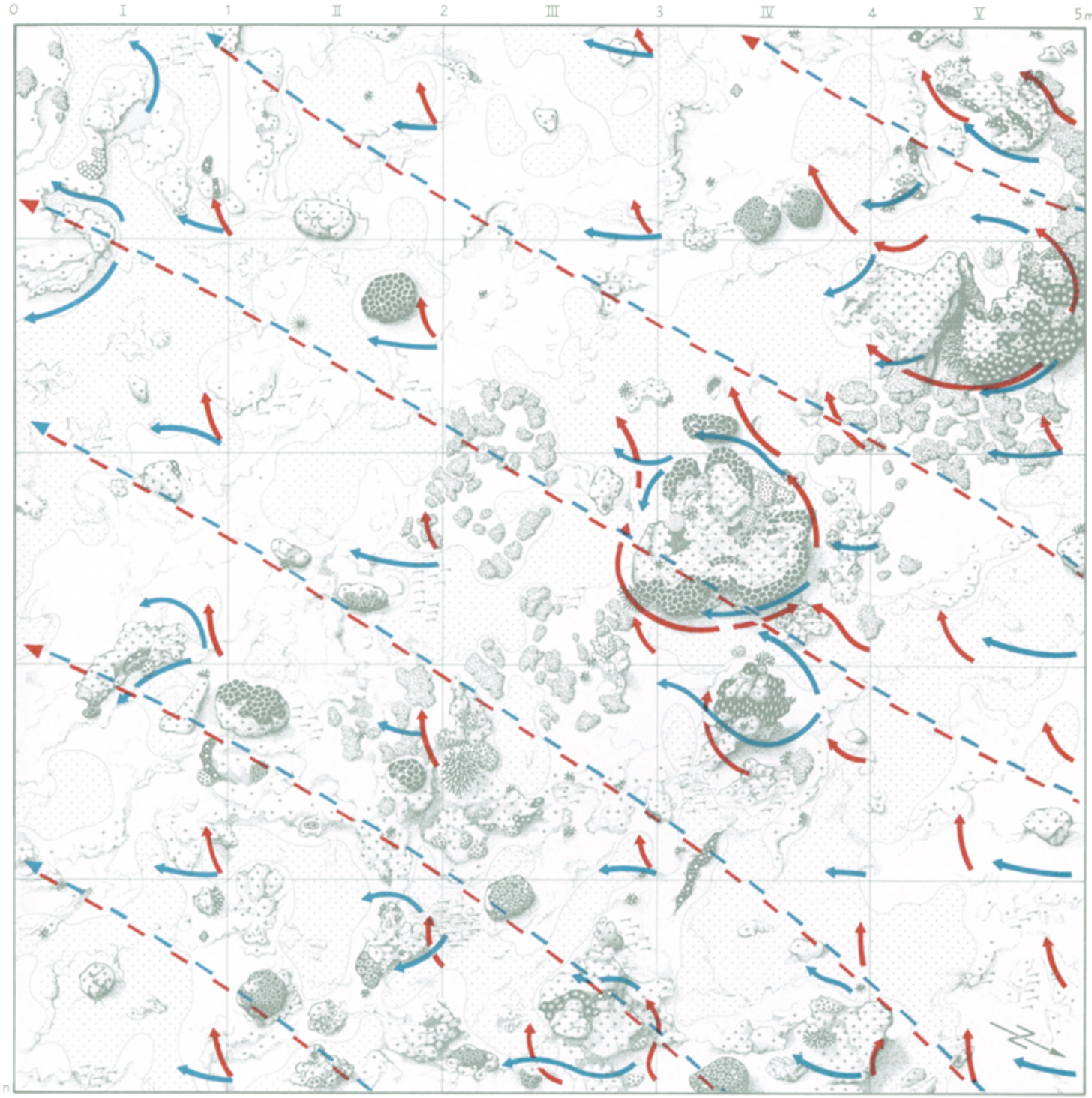

Abb. 2: Strömungsverlauf im Testareal L-1 im Februar 1976, jeweils 1 Std. vor und nach Niedrigwasser. Unterbrochene Pfeile: Oberflächenströmung. Durchzogene Pfeile: Bodennahe Strömung. Blau: Auflaufendes Wasser. Rot: Ablaufendes Wasser 
sommerlichen Zusammenbruch der gesamten Vegetation überdauert und sind auf Protokollphotographien der gleichen Bodenareale vom Herbst und Frühjahr eindeutig mit den neu ausschlagenden Algenkolonien in Verbindung zu bringen. Die Reihenfolge des Erscheinens der einzelnen Algenarten im Testareal und ihre saisonbedingte Entwicklung bis zum Zusammenbruch im Frühsommer sind bei Mergner \& Svoboda (1977) in Fig. 4 graphisch dargestellt.

Tabelle 2 zeigt den Stand der Algenentwicklung im Frühjahr 1976 mit Habitatpräferenz, Saisonalität, Kolonieanzahl und Flächenanteil der Einzelarten in \% der Arealfläche bzw. der Gesamtalgenbedeckung, Abbildung 3 die Verteilung der Arten im Testareal. Gerade diese Abbildung hebt die Braunalge Sargassum dentifolium auch optisch als eindeutig dominierende Leitart der gleichnamigen biophysiographischen Zone hervor wie auch die beiden nächsthäufigen Arten Stypopodium zonale und Dictyota indica. Die Abundanzdaten in Tabelle 2 sichern diesen zunächst subjektiven Eindruck auch quantitativ ab. Lediglich für das in geschlossenen Beständen wachsende Sargassum dentifolium konnte die Kolonieanzahl nur annäherungsweise ermittelt werden. Insgesamt sind im Februar 1976 etwa $26 \%$ der Testarealfläche von Algen überwachsen.

\section{Cnidaria}

Bei der quantitativen Analyse von Schwund und Neuansiedlung der CnidarierKolonien im Winterhalbjahr 1975/76 sowie der jeweiligen prozentualen Anteile an der Gesamtarealfläche (Tab. 3, Abb. 1, 3 und 4) können die beiden Einzelvorkommen von Cerianthus sp. und Tubipora musica mit einer Flächenbedeckung von jeweils unter 0,01\% praktisch vernachlässigt werden. Nach einer Gesamtübersicht sollen zunächst die Hartsubstrat bildenden Hydro- und Steinkorallen analysiert werden, unter denen die Zweigkoralle Stylophora pistillata wiederum eine gesonderte Betrachtung verlangt, und dann die sog. Weichkorallen (Stolonifera, Alcyonaria) einschließlich der Zoantharia.

Insgesamt wurden im Oktober 1975262 Kolonien lebender Cnidarier mit einem Anteil von 6,48 \% der Gesamtarealfläche festgestellt. Unter ihnen waren 16 Kolonien von Millepora exaesa mit 0,22\%, 83 Steinkorallenkolonien mit 2,94\% und 161 Weichkorallenkolonien mit 3,31\%. Von diesem Herbstbestand verschwanden bis März 1976 aus verschiedenen Gründen 35 Kolonien mit 0,46 \% Flächenanteil, vorwiegend Xenia spp. (22 Kolonien), Sinularia polydactyla (6) und Stylopbora pistillata (6). Die Winterverluste wurden jedoch durch Neuansiedlung von 127 jungen Kolonien mit 0,58\% Flächenanteil wieder ausgeglichen, darunter allein Stylophora pistillata mit 65 Kolonien, Sinularia polydactyla mit 7 und Xenia spp. mit 32.

Daraus ergibt sich für März 1976 eine Gesamtabundanz von 354 Kolonien (Zuwachs: $31,3 \%$ ) mit einer prozentualen Flächenbedeckung von 6,60 \% (Zuwachs: 1,7\%). Hiervon entfielen auf Millepora 23 Kolonien mit 0,32\% Flächenanteil (Zuwachs: 43,8 bzw. 44,1\%), auf Steinkorallen 148 Kolonien mit 3,09 \% Fläche (Zuwachs: 78,3 bzw. 20,2 \%) und auf Weichkorallen 176 Kolonien mit 3,18 \% Fläche (9,3 \% Zuwachs der Kolonieanzahl bzw. 2,6 \% Abnahme des Flächenanteils). Auffallend ist der mit 0,12\% nur sehr geringe Nettoflächenzuwachs aller Cnidarier trotz erheblicher Zunahme ihrer Kolonieanzahl um 92. Dabei resultiert dieser Nettozuwachs vor allem aus der fast $100 \%$ igen Vermehrung der Stylophora-Kolonien bei einem Flächenzuwachs von 20,0\% und aus der 


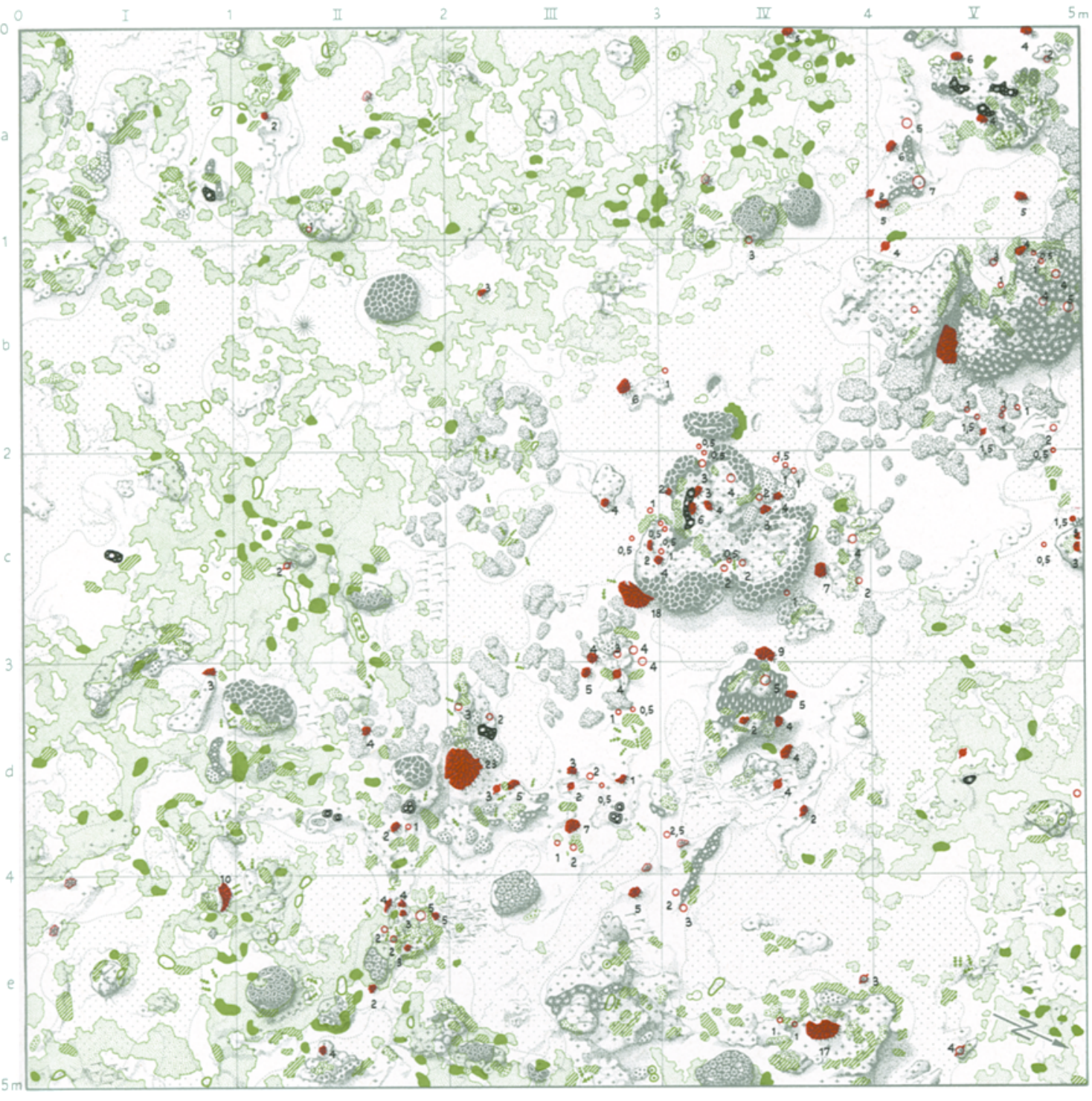

Algae
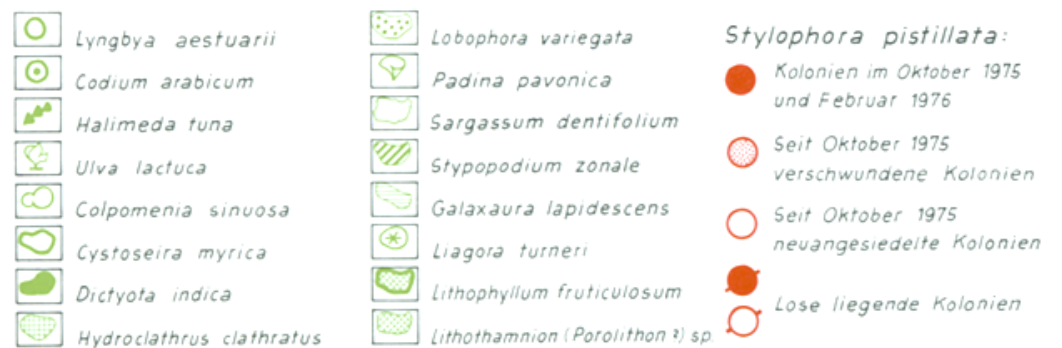

Neuansiedlung

anderer Arten:

\&

8

용

Abb. 3: Verteilung der benthischen Makroalgen und der Zweigkoralle Stylophora pistillata im Testareal L-1 (Februar 1976) mit saisonalen Abundanzänderungen der Hydro- und Steinkorallen (Cnidaria-1) von Oktober 1975 bis Februar 1976 


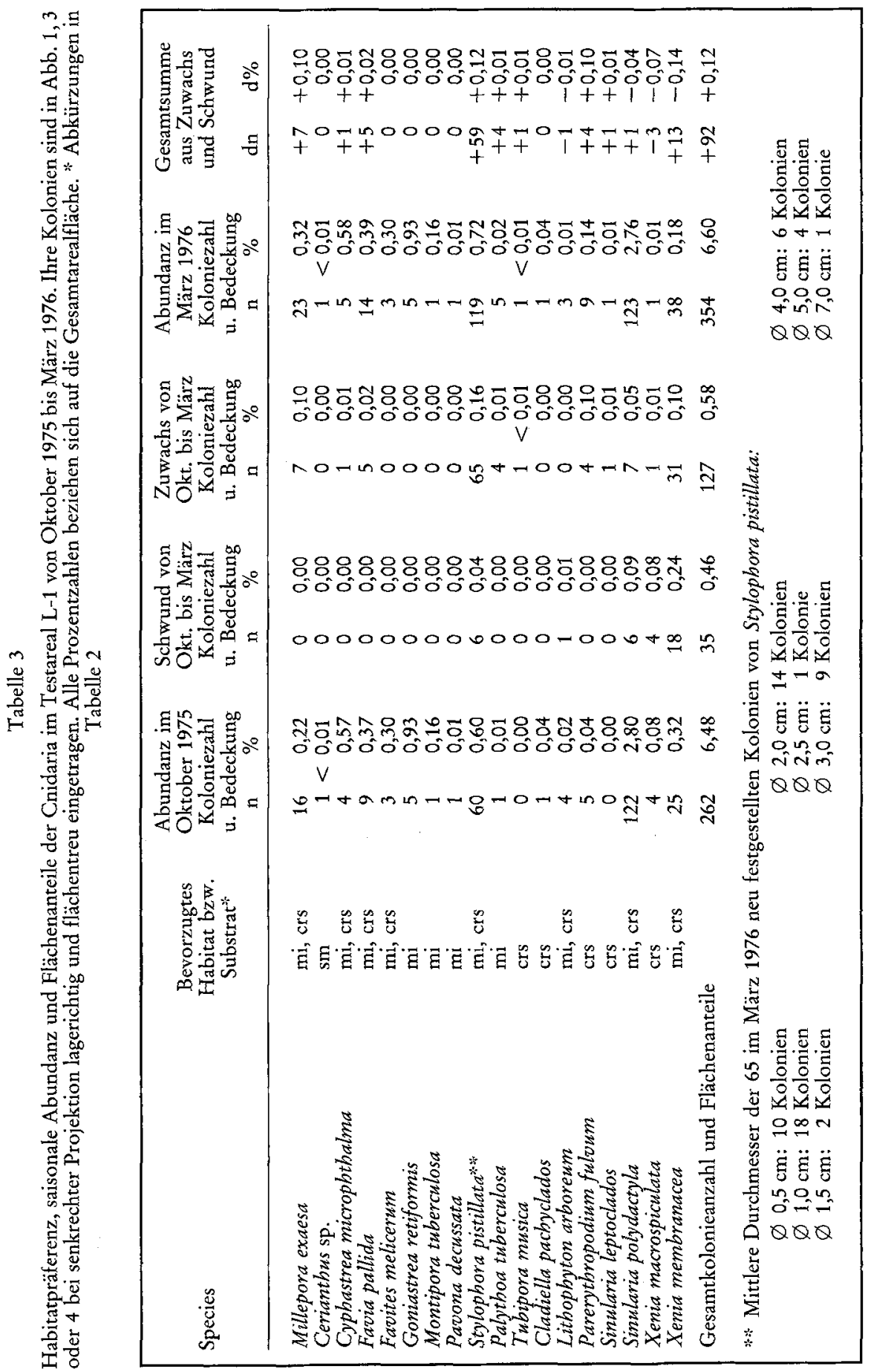




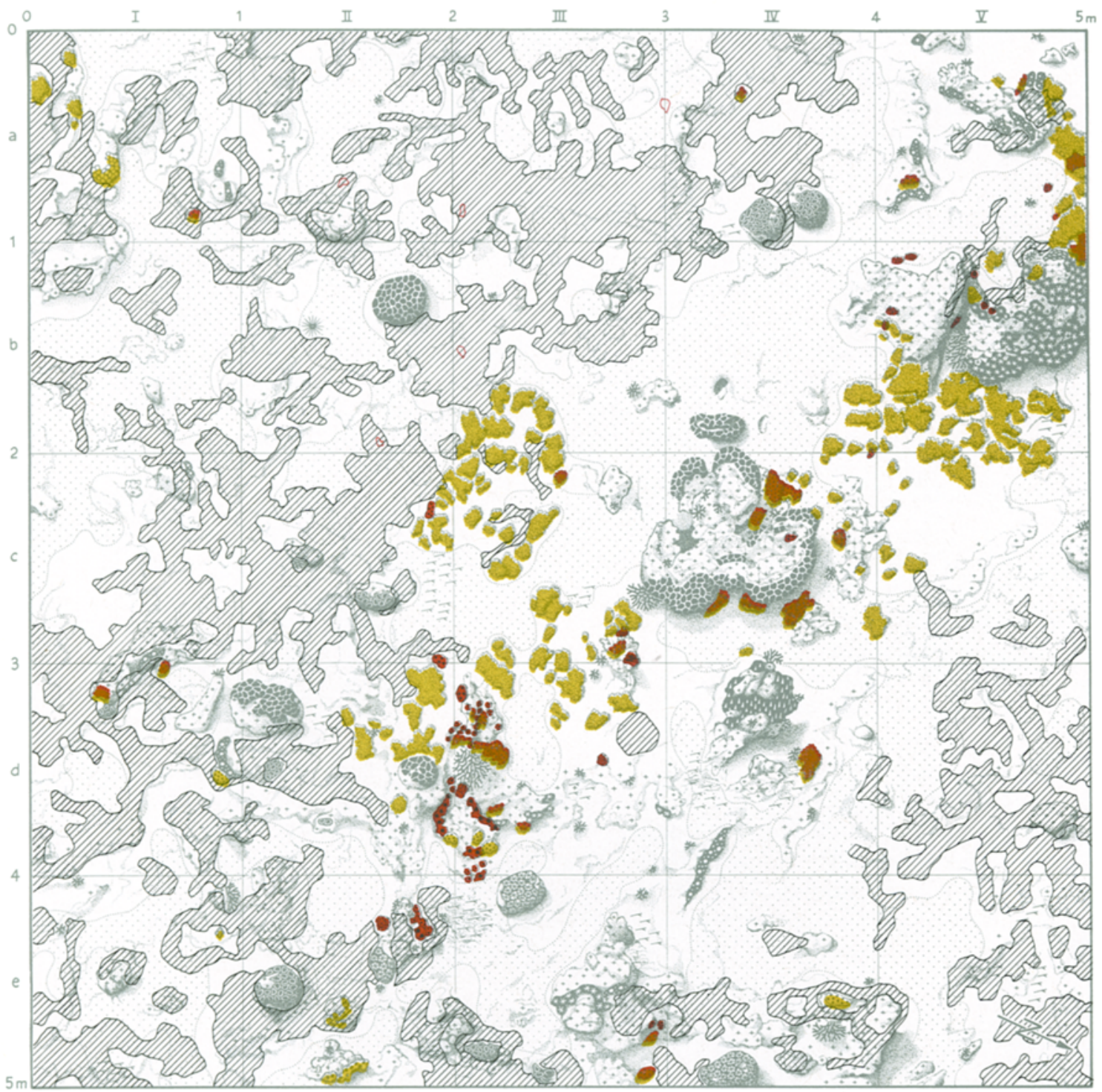

Cnidaria - 2

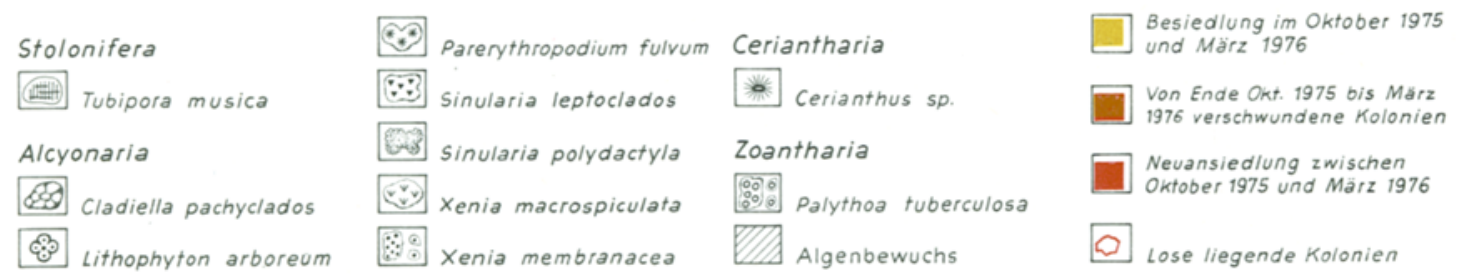

Abb. 4: Verteilung und saisonale Abundanzänderungen der Weichkorallen (Cnidaria-2) im Testareal L-1 von Oktober 1975 bis Februar 1976 
von Parerythropodium fulvum (Zuwachs: 80,0 bzw. 250,0\%). Daher ist anzunehmen, dal3 die meisten der neu angesiedelten winzigen Kolonien nach kürzester Zeit wieder absterben und nur höchstens $10 \%$ von ihnen bis zu einem Jahr überleben.

\section{Hydro- und Steinkorallen}

Von der Zweigkoralle Stylophora pistillata abgesehen, waren die saisonalen Abundanzveränderungen bei Hydro- und Steinkorallen während des Winterhalbjahres 1975/76 gering. Erkennbare Verluste traten nicht ein; allerdings blieb auch der Umfang der Neuansiedlungen begrenzt: Einige der 7 neu aufgefundenen Kolonien von Millepora exaesa waren möglicherweise schon im Herbst vorhanden, aber wegen ihrer Bodennähe von Sandmulm überdeckt. Nachweislich neu angesiedelt haben sich aber 5 kleine, aus nur 1-3 Polypen bestehende Kolonien von Favia pallida und eine von Cyphastrea microphthalma (Tab. 3, Abb. 3).

Im Gegensatz zu diesen massig wachsenden Skleraktinien-Arten zeigte die Zweigkoralle Stylophora pistillata eine bemerkenswerte Zuwachsrate (Tab. 3, Abb. 1 und 3): Nach dem Verlust von $10 \%$ der im Oktober 1975 festgestellten Kolonien verdoppelte sich ihr Bestand bis März 1976. Da die Brutsaison dieser Art von Oktober bis in den Frühsommer hinein dauert, finden sich während dieser Zeit ständig Neuansiedlungen mit entsprechend unterschiedlichen Durchmessern. In Tabelle 3 sind die 54 gezählten Neukolonien von 0,3 bis $3,0 \mathrm{~cm}$ aufgeschlüsselt und in Abbildung 3 kartiert. Bei den übrigen 11 von 4,0 bis $7,0 \mathrm{~cm}$ großen Kolonien könnte es sich um abgebrochene und wieder festgesetzte Teile größerer Stöcke handeln, wie dies bei Stylophora unter günstigen Umständen möglich sein soll. Von der Besiedlung bleibt das geschlossene Algenareal fast völlig frei (Abb. 3): Im Oktober 1975 fanden sich in ihm nur 7 Kolonien, von denen im Winterhalbjahr 4 verschwanden, während 3 neue hinzukamen. Möglicherweise werden die StylophoraLarven durch den dichten Algenwuchs am Festsetzen gehindert. Als Anheftungsflächen werden offensichtlich Hartsubstrate benötigt. Die beiden einzigen auf reinen Sandmulmflächen gefundenen Kolonien waren nämlich auch mit ihrer Basis an halb eingesandeten Korallenbruchstïcken festgewachsen.

\section{Weichkorallen}

Unter den saisonalen Abundanzveränderungen der Weichkorallen fällt vor allem das Verschwinden von 22 Xenia-Kolonien (76\%) im Umkreis der beiden Mikroatolle und einiger größerer Korallenhorste auf, dem ein Zuwachs von nicht weniger als 31 Neukolonien $(107 \%)$ mit Durchmessern von $1-3 \mathrm{~cm}$ gegenübersteht. Nahezu alle von ihnen haben sich in unmittelbarer Nachbarschaft früherer Xenia membranacea - Stöcke angesiedelt (Tab. 3, Abb. 1 und 4). Beide Beobachtungen werden später noch diskutiert (vgl. p. 504).

Bemerkenswert war auch die rasche Flächenausbreitung von Parerytbropodium fulvum während der Untersuchungszeit, in der sich ihr Areal mehr als verdreifachte, und erwähnenswert die Ansiedlung je einer kleinen Kolonie von Tubipora musica und Sinularia leptoclados in diesem für sie untypischen Habitat. Während bei Sinularia polydactyla der Verlust von 6 Kolonien durch 7 neu hinzugekommene bei annähernd gleicher Flächenbedeckung wieder ausgeglichen wurde, ergaben sich bei den übrigen Weichkorallen keine 


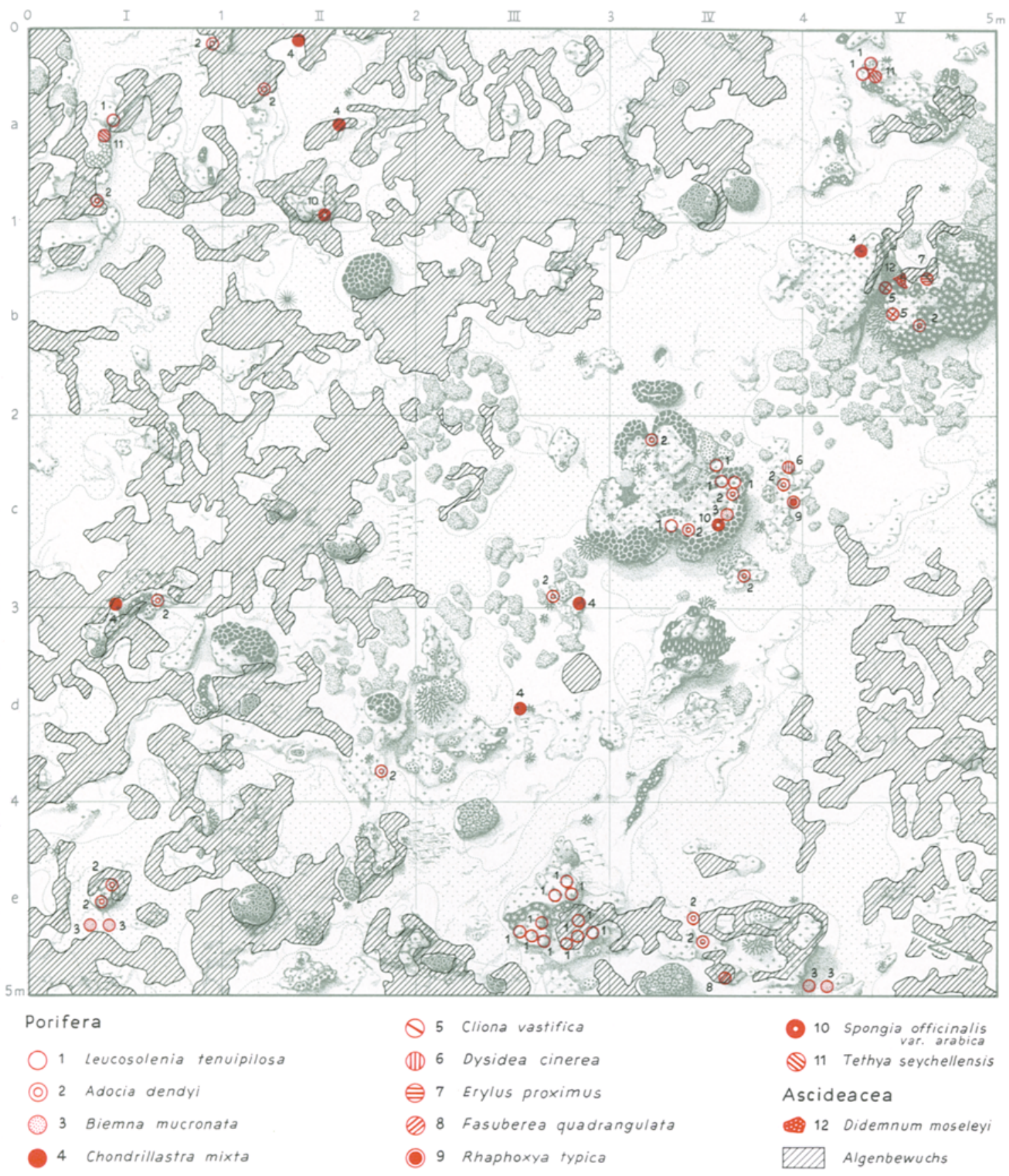

Abb. 5: Verteilung kleiner sessiler Tiergruppen (Porifera, Ascidiacea) im Testareal L-1 im Februar 1976 
oder nur geringfügige Veränderungen. Bei der Zoantharie Palythoa tuberculosa fanden sich dagegen im März 1976 in unmittelbarer Nähe der einzigen Altkolonie 4 winzige, meist nur aus einem Polypen bestehende Neukolonien, die aber den Flächenanteil dieser Art nur unwesentlich vergrößerten.

\section{Kleinere Tiergruppen}

Eine Anzahl kleinerer sessiler und mobiler Tiergruppen wurde nur der Vollständigkeit halber in die Untersuchungen zu Abundanz, Habitatpräferenz und Artendiversität der Testarealbesiedlung miteinbezogen, da ihre meist nur geringe Individuenzahl keine quantitativen Aussagen zuließ. Dies gilt vor allem für Turbellarien, errante und sedentäre Polychäten sowie die einzige im Untersuchungsareal angetroffene Ascidienkolonie von Didemnum moseleyi. Zwar ist von den makrobenthischen Foraminiferenarten Homotrema rubrum massenhaft unter Korallenfelsstrukturen zu finden, doch lassen sich ihre sehr unterschiedlich gestalteten Gehäuse nur mit rasterelektronenoptischen Methoden von denen der äußerlich gleich aussehenden Miniacina miniacea unterscheiden. Ebenfalls ganzjährig, aber nicht so häufig, kommt im gleichen Habitat und nicht selten an Makroalgen die nummulitenähnliche Maginopora vor. Eine vollständige quantitative Abundanzanalyse der Foraminiferen würde freilich ebenso wie die der Serpulide Pileolaria militaris die weitgehende Zerstörung der im Testareal vorhandenen Hartsubstratstrukturen erfordern. Weitere Informationen über die oben genannten Gruppen sind den Tabellen 4 und 5 sowie den Abbildungen 5 und $6 \mathrm{zu}$ entnehmen. Im folgenden sollen daher von den kleineren sessilen Tiergruppen nur die Poriferen und von den kleineren mobilen Gruppen nur die Crustaceen gesondert behandelt werden, von denen jeweils eine größere Anzahl Arten kartiert werden konnte.

\section{Porifera}

Die Poriferen zeigten innerhalb des Testareals L-1 die erwartete charakteristische Bindung an mehr oder weniger stark beschattete Hartsubstrate wie die beiden Mikroatolle, einige erodierte Korallenfelshorste und die Unterseite einer großen, lose liegenden Bodenplatte (Abb. 5). Lediglich Biemna mucronata fand sich auf hell beleuchteten Sandmulden. Unerwartet hoch war dagegen die Besiedlungsdichte mit insgesamt 11 Arten und 65 Kolonien für einen derart begrenzten und einförmigen Lagunenbereich. Freilich kamen nur Leucosolenia tenuipilosa bei streng kryptischer Lebensweise unter der erwähnten Bodenplatte, Adocia dendyi und der Bohrschwamm Cliona vastifica in größerer Kolonieanzahl vor (Tab. 4). F. Nobbe (in Vorbereitung) wird über Faunistik und Ökologie der Poriferenbestände in der Rifflagune noch ausführlich berichten.

\section{Crustacea}

Um vorzeitige Störungen der Besiedlungsstrukturen im Testareal L-1 zu vermeiden, wurde eine quantitative Abundanzanalyse der Crustaceenfauna erst im Februar 1976 nach Aufkommen des Algenwuchses vorgenommen. Dabei wurden insgesamt 47 Individuen aus 22 Arten gefunden und von letzteren 17 kartiert (Tab. 5, Abb. 6). Tatsächlich dürfte aber 


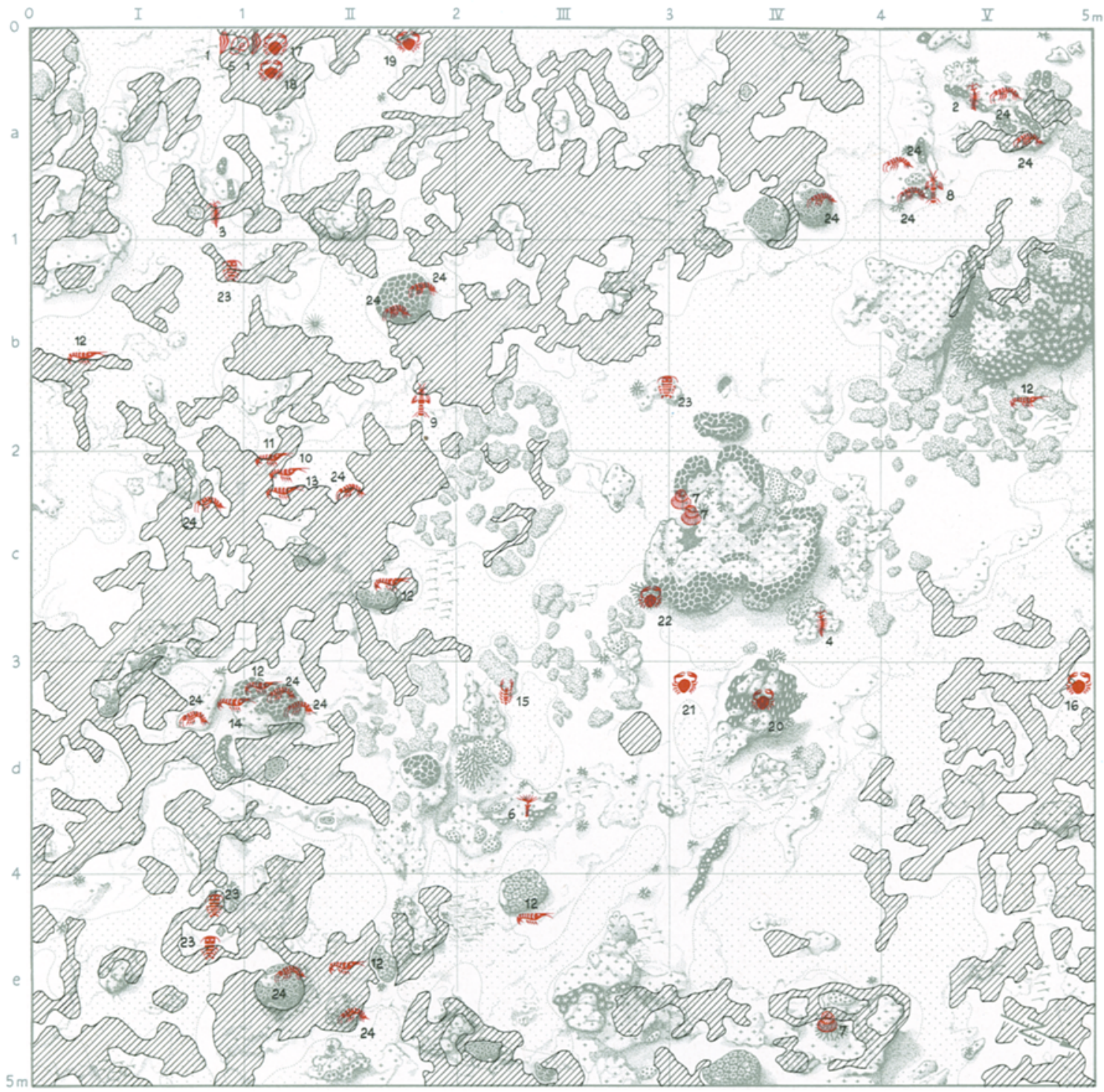

Turbellaria

1 stylochidae

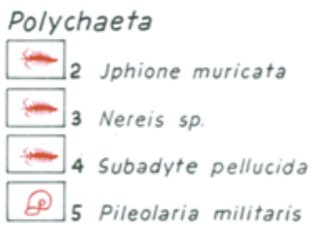

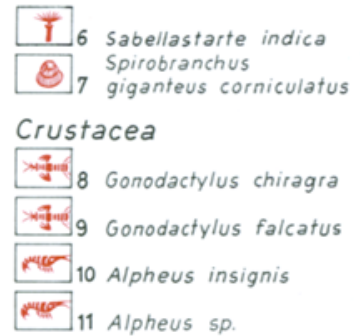

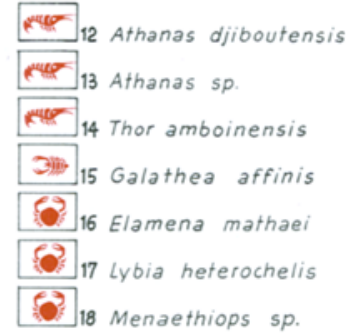

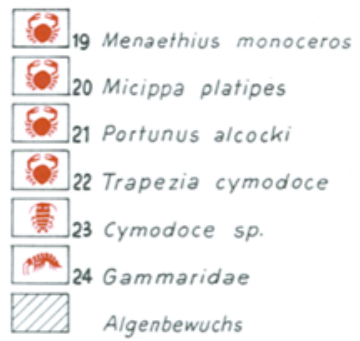

Abb. 6: Verteilung kleiner mobiler Tiergruppen (Turbellaria, Polychaeta, Crustacea) im Testareal L-1 im Februar 1976 
die Besiedlung, vor allem bei den kryptisch lebenden Arten, noch dichter sein, als dies aus der geringen Individuenzahl der Einzelarten hervorgeht. Bekannte Habitatbindungen fanden ihre Bestätigung, etwa die taphobiontischer Natantia an sandmulmgefüllte Mulden bzw. an Phytalflächen oder die von Trapezia cymodoce und mancher Alpheiden an die Zweigkoralle Stylophora pistillata. Ein Zusammenhang zwischen der raschen Entwicklung der Algenbestände und einer gleichzeitigen Verdichtung der Crustaceenbesiedlung, etwa durch Zuwanderung aus benachbarten Lagunenbereichen, konnte nicht gesichert werden, auch wenn seit Oktober 1975 eine augenscheinliche Zunahme der Arten- und Individuenzahlen erfolgt ist.

\section{Mollusca}

Die mehrfach wiederholte Bestandsaufnahme der Molluskenfauna im Bereich der Rifflagune (Mastaller, in Vorbereitung) erlaubt nunmehr eindeutige Aussagen zur Populationsdynamik und Artendiversität dieser Gruppe und einen Vergleich mit den Daten aus anderen Riffzonen: Die erste Aufsammlung von Mollusken im Testareal L-1 im Februar 1976, also in einer Zeit üppigster Algenentwicklung, erbrachte 477 Individuen (davon 7 als tote Schalen) aus 60 Arten, die in Abbildung 7 lagerichtig kartiert sind. Im Juni 1976 wurden noch 378 Individuen aus 65 Arten und im November 1977 noch 292 Individuen aus 75 Arten festgestellt (Tab. 6). Insgesamt wurden also auf einer Testfläche von nur $25 \mathrm{~m}^{2}$ 113 Arten (105 lebend) mit 1147 Individuen gefunden. Diese Zahlen demonstrieren eine

Tabelle 4

Habitatpräferenz und Abundanz kleiner sessiler Tiergruppen im Testareal L-1 (Frühjahr 1976). Die Arten Nr. 1-5, 7-9, 11 und 12 wurden am 9. Februar kartiert, Nr. 6 und 10 am 27. Mai 1976. Alle diese Arten sind mit gleichen Nummern in Abb. 5 bei senkrechter Projektion lagerichtig eingetragen. Von den Foraminiferen bestehen dagegen keine Fundortangaben. * Abkürzungen in Tabelle 2

\begin{tabular}{|c|c|c|}
\hline Species & $\begin{array}{c}\text { Bevorzugtes } \\
\text { Habitat bzw. } \\
\text { Substrat* }\end{array}$ & $\begin{array}{l}\text { Anzahl der } \\
\text { Individuen } \\
\text { bzw. Kolonien }\end{array}$ \\
\hline $\begin{array}{l}\text { For a min if e ra } \\
\text { Homotrema cf. rubrum } \\
\text { Maginopora sp. }\end{array}$ & $\begin{array}{l}\text { mi, crs } \\
\text { phy, crs }\end{array}$ & $\begin{array}{l}>200 \\
>30\end{array}$ \\
\hline $\begin{array}{l}\text { Porif e r a } \\
\text { 1. Leucosolenia tenuipilosa } \\
\text { 2. Adocia dendyi } \\
\text { 3. Biemna mucronata } \\
\text { 4. Chondrillastra mixta } \\
\text { 5. Cliona vastifica } \\
\text { 6. Dysidea cinerea } \\
\text { 7. Erylus proximus } \\
\text { 8. Fasuberea quandrangulata } \\
\text { 9. Rhaphoxya typica } \\
\text { 10. Spongia officinalis, var, arabica } \\
\text { 11. Tethya seychellensis }\end{array}$ & $\begin{array}{l}\mathrm{mi}, \mathrm{crs} \\
\mathrm{mi}, \mathrm{crs} \\
\text { sm } \\
\mathrm{crs} \\
\mathrm{mi} \\
\mathrm{crs} \\
\mathrm{mi} \\
\mathrm{crs} \\
\mathrm{crs} \\
\mathrm{mi} \\
\mathrm{crs}\end{array}$ & $\begin{array}{r}18 \\
16 \\
5 \\
6 \\
12 \\
1 \\
1 \\
1 \\
1 \\
2 \\
2\end{array}$ \\
\hline Gesamtkolonieanzahl der Porifera & & 65 \\
\hline $\begin{array}{l}\text { Ascidiace a } \\
\text { 12. Didemnum moseleyi }\end{array}$ & $\mathrm{mi}$ & 1 \\
\hline
\end{tabular}




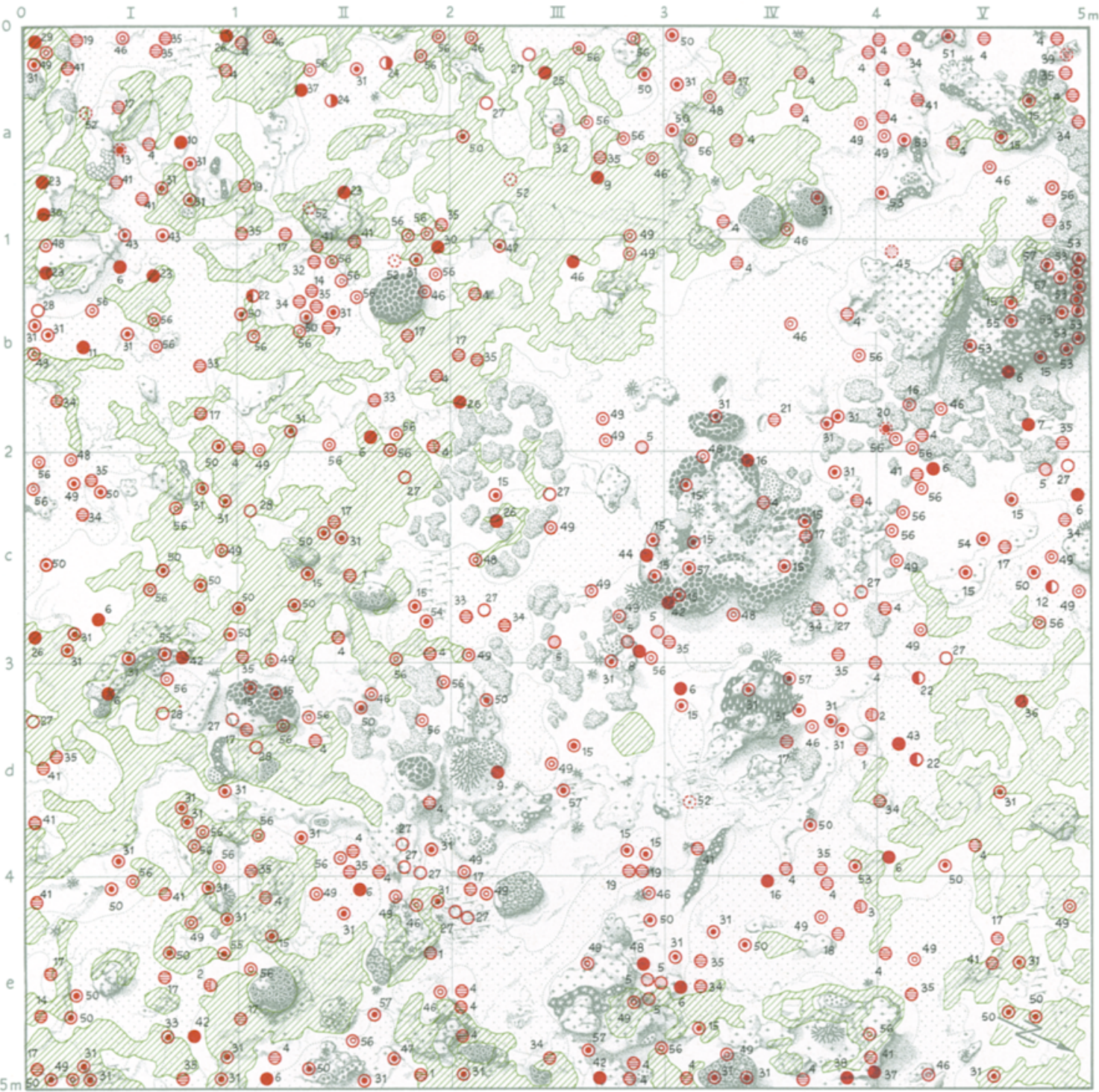

Mollusca Ernährungsweise
$\theta$ Herbivor
Carnivor
(C) Mobile Filtrierer
Algenbewuchs
(1) Herbivor / Detritophag
D Carnivor / Nekrophag
- Sessile Filtrierer
- Detritophag
Nekrophag
毫 (-) Nur als Schalen gefunden

Abb. 7: Verteilung und Ernährungsweise der Mollusken im Testareal L-1 im Februar 1976. Die Zahlen an den Symbolen beziehen sich auf die unter den gleichen Nummern verzeichneten Artnamen in Tab. 6 
eindrucksvolle Arten- und Individuendichte. Zugleich beinhalten sie ein Fünftel aller bisher aus dem Roten Meer beschriebenen Arten (Mastaller, 1978). Darüber hinaus weisen sie auf einen hohen Grad der Artendiversität hin (Tab. 9). Mit einem Index H = 3,321 bis 3,673 übertrifft er den benachbarter Riffzonen wie der reinen Sargassum dentifolium Zone des Ufersaumriffes $(\mathrm{H}=2,492)$ oder der Halodule uninervis - Zone des Lagunensaumriffes $(\mathrm{H}=2,582)$ bei weitem (Mergner \& Mastaller, 1979 im Druck).

Abbildung 7 zeigt für Februar 1976 die Einzelfundplätze der herbivoren Arten nicht ausschließlich im Bereich dichter Algenbedeckung: Hier wurden von 141 Herbivoren und Detritophagen nur 70 festgestellt, während sich weitere 22 auf algenüberwachsenem Korallenfels fanden und der Rest auf algenfreie Bodenplatten und Sandmulden aufteilte.

Tabelle 5

Habitatpräferenz und Abundanz kleiner überwiegend mobiler Tiergruppen im Testareal L-1 (Frühjahr 1976). Die Arten Nr. 1-24 wurden am 21. Februar 1976 kartiert und mit gleichen Nummern in Abb. 6 bei senkrechter Projektion lagerichtig eingetragen. Von den anderen Arten bestehen keine Fundortangaben. * Abkürzungen in Tabelle 2

\begin{tabular}{|c|c|c|}
\hline Species & $\begin{array}{c}\text { Bevorzugtes } \\
\text { Habitat bzw. } \\
\text { Substrat:* }\end{array}$ & $\begin{array}{l}\text { Anzahl der } \\
\text { Individuen }\end{array}$ \\
\hline \multicolumn{3}{|l|}{ Turbellaria } \\
\hline $\begin{array}{l}\text { Poly cha t a } \\
\text { 2. lpbione muricata } \\
\text { 3. Nereis sp. } \\
\text { 4. Subadyte pellucida } \\
\text { 5. Pileolaria militaris } \\
\text { 6. Sabellastarte indica } \\
\text { 7. Spirobrancbus giganteus corniculatus }\end{array}$ & $\begin{array}{l}\mathrm{sm}, \mathrm{crs} \\
\mathrm{sm}, \mathrm{crs} \\
\mathrm{sm}, \mathrm{crs} \\
\mathrm{mi}, \mathrm{crs} \\
\mathrm{crs} \\
\mathrm{mi}, \mathrm{crs}\end{array}$ & $\begin{array}{r}1 \\
2 \\
1 \\
>100 \\
1 \\
3\end{array}$ \\
\hline \multicolumn{3}{|l|}{ Crustacea } \\
\hline 8. Gonodactylus cbiragra & crs & 1 \\
\hline 9. Gonodactylus falcatus & $\mathrm{sm}$ & 1 \\
\hline 10. Alpheus insignis & phy, sm & 1 \\
\hline 11. Alpheus sp. & phy, sm & 1 \\
\hline 12. Athanas djiboutensis & phy, sm, mi, crs & 6 \\
\hline 13. Athanas sp. & $\mathrm{sm}$ & 1 \\
\hline 14. Thor amboinensis & $\mathrm{sm}, \mathrm{mi}$ & 1 \\
\hline Calcinus latens & & 1 \\
\hline Clibanarius signatus & & 1 \\
\hline 15. Galathea affinis & $\mathrm{sm}$ & 1 \\
\hline Petrolisthes leptocheles & & 1 \\
\hline Pisidia inaequalis & & 1 \\
\hline 16. Elamena mathaei & crs & 1 \\
\hline 17. Lybia beterochelis & crs & 3 \\
\hline Medaeus neglectus & & 1 \\
\hline 18. Menaethiops sp. & crs & 2 \\
\hline 19. Menaethius monoceros & $\mathrm{sm}$ & 1 \\
\hline 20. Micippa platipes & $\mathrm{mi}$ & 1 \\
\hline 21. Portunus alcocki & $\mathrm{sm}$ & 2 \\
\hline 22. Trapezia cymodoce & Stylophora & 1 \\
\hline 23. Cymodoce sp. & phy, sm & 4 \\
\hline 24. Gammaridae & $\mathrm{sm}, \mathrm{mi}, \mathrm{crs}$ & 14 \\
\hline Gesamtindividuenanzahl der Crustacea & & 47 \\
\hline
\end{tabular}




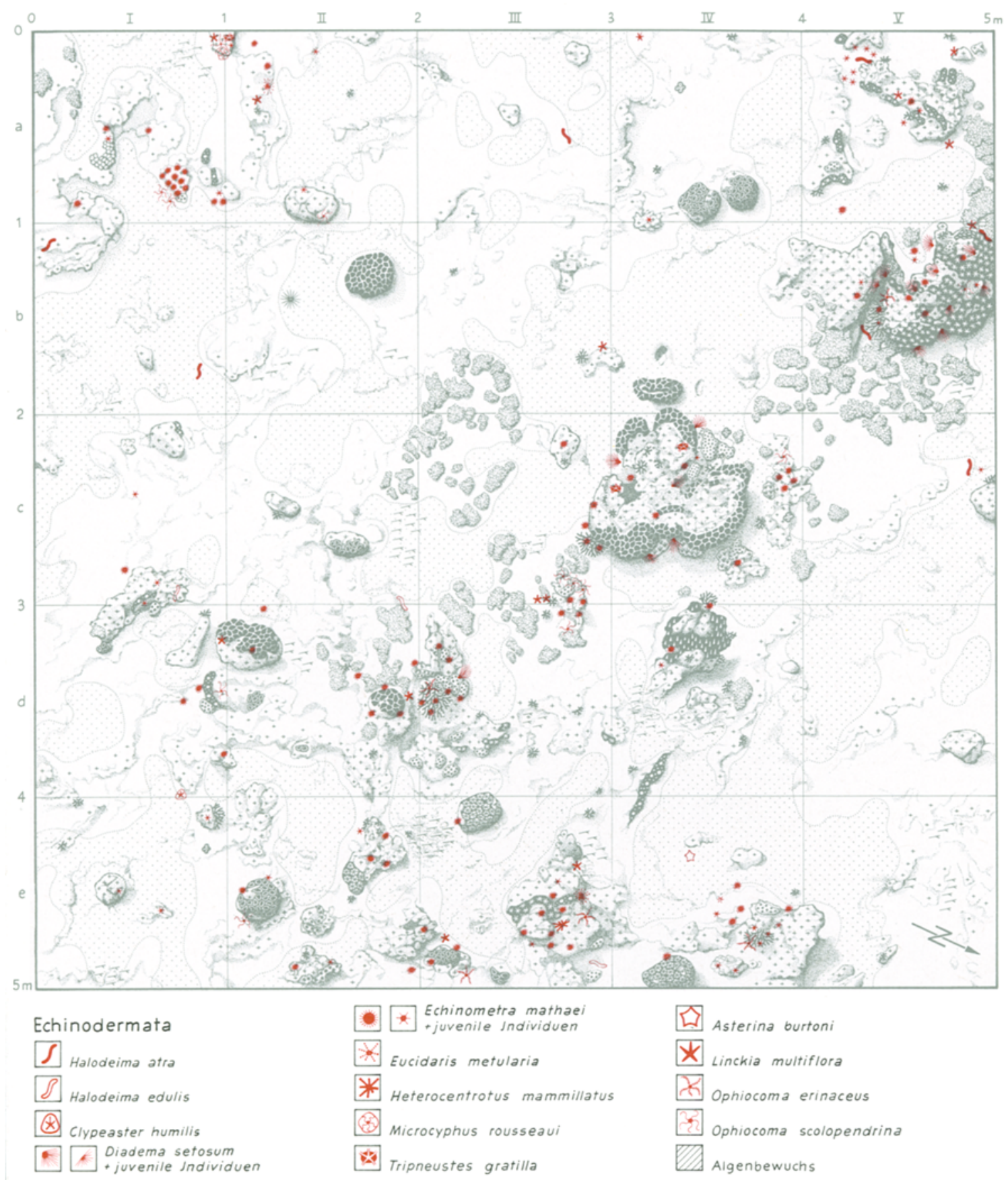

Abb. 8: Verteilung der Echinodermen im Testareal L-1 im Februar 1976 
Tabelle 6

Ennährungsweise, Habitatpräferenz und saisonale Abundanz der Mollusca im Testareal L-1. Die Arten Nr. 1-57 wurden vom 7. bis 12 . Februar 1976 kartiert und mit gleichen Nummern in Abb. 7 bei senkrechter Projektion lagerichtig eingetragen. Die anderen Arten und Individuen wurden vom 2. bis 5. Juni 1976 und vom 6. bis 8. November 1977 gesammelt, aber nicht kartiert. + - Nur als Schalen gefunden. * $\mathrm{sM}$ - Sessile Leimrutenfänger; $\mathrm{sF}$ - Sessile Filtrierer; $\mathrm{mF}$ - Mobile Filtrierer; $\mathrm{sP}$ - Sessile Parasiten; P - Parasiten; C - Carnivore Arten; D - Detritophage Arten; H - Herbivore Arten; NNekrophage Arten. $*$ Abkürzungen in Tabelle 2. $* *$ Arten mit vermuteter Tendenz zur Abwanderung in andere Riffzonen nach der Phytalregression im Frühsommer, so in die: * Halodule uninervis -Zone; o-Mikroatollzone

\begin{tabular}{|c|c|c|c|c|c|c|}
\hline Species & $\begin{array}{l}\text { Ernäh- } \\
\text { rungs- } \\
\text { weise* }\end{array}$ & $\begin{array}{l}\text { Bevorzugtes } \\
\text { Habitat bzw. } \\
\text { Substrat } * \%\end{array}$ & $\begin{array}{l}\text { Saisonale } \\
\text { Abwande- } \\
\text { rung in } * * \%\end{array}$ & $\begin{array}{l}\text { Anz. d. I } \\
\text { d. Unter } \\
\text { Febr. } 76\end{array}$ & $\begin{array}{l}\text { Individue } \\
\text { suchungs } \\
\text { Juni } 76\end{array}$ & $\begin{array}{l}\text { en währ. } \\
\text { sper. im } \\
\text { Nov. } 77\end{array}$ \\
\hline 1. Ischnocbiton yerburi & $\mathrm{H}$ & $\mathrm{mi}$ & & 6 & 7 & 3 \\
\hline Tonicia costata & $\mathrm{H}$ & $\mathrm{mi}$ & & & 1 & \\
\hline Tonicia suezensis & $\mathrm{H}$ & crs & & & 1 & 1 \\
\hline Ancilla lineolata & C & $\mathrm{sm}$ & & & & 1 \\
\hline Apollon pusillus & C & $\mathrm{mi}$ & & & & 1 \\
\hline Balcis cf. acuta & $\mathrm{P}$ & $\mathrm{sm}$ & & & & 1 \\
\hline 2. Cerithium caeruleum & $\mathrm{H}, \mathrm{D}$ & phy, sm crs & & 2 & & 3 \\
\hline 3. Ceritbium columna & $\mathrm{H}, \mathrm{D}$ & phy, crs & & 1 & 1 & 2 \\
\hline $\begin{array}{l}\text { Cerithium nesioticum } \\
\text { 4. Cerithium nodulosum }\end{array}$ & $\mathrm{H}, \mathrm{D}$ & $\mathrm{mi}, \mathrm{crs}$ & 0 & & 1 & 2 \\
\hline erythraeonense & $\mathrm{H}$ & phy & $*$ & 45 & 17 & 14 \\
\hline 5. Cerithium rueppelli & $\mathrm{D}$ & $\mathrm{sm}$ & & 8 & 8 & 2 \\
\hline Cerithium scabridum & $\mathrm{H}, \mathrm{D}$ & $\mathrm{sm}$ & & & & 1 \\
\hline Clypeomorus tuberculatus & $\mathrm{H}, \mathrm{D}$ & crs & $*$ & & 4 & 1 \\
\hline Conus arenatus & C & $\mathrm{sm}$ & $*$ & & 5 & \\
\hline 6. Conus coronatus & C & phy, sm, crs & & 12 & 3 & 4 \\
\hline 7. Conus lividus & C & $\mathrm{sm}$ & & 1 & 1 & \\
\hline 8. Conus spirogloxus & C & phy, sm & & 1 & & \\
\hline Conus striatus + & $\mathrm{C}$ & $\mathrm{sm}$ & & & 1 & \\
\hline 9. Conus taeniatus & $\mathrm{C}$ & $\mathrm{sm}, \mathrm{crs}$ & & 3 & 8 & 1 \\
\hline 10. Conus tessulatus & $\mathrm{C}$ & $\mathrm{sm}$ & & 1 & 3 & \\
\hline 11. Conus textile & $\mathrm{C}$ & phy, sm & & 1 & & \\
\hline Coralliophilia violacea & $\mathrm{sP}$ & $\mathrm{mi}$ & & 1 & & 1 \\
\hline 12. Cymatium pileare & $\mathrm{C}, \mathrm{N}$ & crs & & 1 & & \\
\hline 13. Cymatium rubecula + & $c$ & $\mathrm{sm}$ & & 1 & & \\
\hline 14. Cypraea arabica & $\mathrm{H}$ & crs & & 1 & & \\
\hline 15. Dendropoma maximum & $\mathrm{sM}$ & $\mathrm{mi}$ & & 24 & 20 & 16 \\
\hline Drupa digitata & $\mathrm{C}$ & $\mathrm{mi}$ & & & & 2 \\
\hline Drupella rugosa & $\mathrm{C}$ & $\mathrm{mi}$ & & & & 1 \\
\hline 16. Fusinus polygonoides & $\mathrm{C}$ & phy, sm & & 2 & & \\
\hline Gena auricularia + & $\mathrm{H}$ & phy & & & & 1 \\
\hline 17. Gena varia & $\mathrm{H}$ & mi, crs & & 19 & 29 & 14 \\
\hline 18. Gibbula declivis & $\mathrm{H}$ & $\mathrm{mi}, \mathrm{crs}$ & $*$ & 1 & 2 & \\
\hline 19. Haliotis pustulata & $\mathrm{H}$ & $\mathrm{mi}, \mathrm{crs}$ & & 4 & 3 & 4 \\
\hline 20. Harpa minor + & $\mathrm{C}$ & $\mathrm{sm}$ & & 1 & 1 & \\
\hline Heliacus variegatus & $\mathrm{P}$ & $\mathrm{mi}$ & & & & 1 \\
\hline Hemitoma tricarinata & $\mathrm{H}$ & phy, crs & & & 2 & 1 \\
\hline Hydatina physis & $\mathrm{H}$ & sm & & & 1 & \\
\hline 21. Lambis truncata sebae & $\mathrm{H}$ & $\mathrm{sm}$ & $*$ & 1 & & \\
\hline Latirus turritus & $\mathrm{C}$ & crs & & & 1 & 1 \\
\hline 22. Maculotriton & & & & & & \\
\hline $\begin{array}{l}\text { cf. serriale } \\
\text { Marginell savionvi }\end{array}$ & $\mathrm{C}, \mathrm{N}$ & $\mathrm{mi}$ & 0 & 3 & 13 & $\begin{array}{r}12 \\
1\end{array}$ \\
\hline $\begin{array}{l}\text { Marginella savignyi } \\
\text { Melampus flavus }\end{array}$ & $\mathrm{H}$ & $\begin{array}{l}\mathrm{ml}, \mathrm{crs} \\
\mathrm{mi}, \mathrm{crs}\end{array}$ & & & 1 & \\
\hline Mitra cucumerina & $\mathrm{C}$ & phy & & & & 1 \\
\hline
\end{tabular}


Tabelle 6 (1. Fortsetzung)

\begin{tabular}{|c|c|c|c|c|c|c|}
\hline Species & $\begin{array}{l}\text { Ernäh- } \\
\text { rungs- } \\
\text { weise: }\end{array}$ & $\begin{array}{l}\text { Bevorzugtes } \\
\text { Habitat bzw. } \\
\text { Substrat }\end{array}$ & $\begin{array}{l}\text { Saisonale } \\
\text { Abwande- } \\
\text { rung in } 1\end{array}$ & $\begin{array}{r}\text { Indivic } \\
\text { d. Unter } \\
\text { Febr. } 76 \\
\end{array}$ & $\begin{array}{l}\text { duenzahl } \\
\text { rsuchungs } \\
\text { Juni } 76\end{array}$ & $\begin{array}{l}\text { währ. } \\
\text { sper. im } \\
\text { Nov. } 77\end{array}$ \\
\hline 23. Mitra fasciolaris & C & crs & 0 & 4 & & 2 \\
\hline 24. Mitra litterata & $\mathrm{N}, \mathrm{C}$ & phy, sm, crs & $\because$ & 2 & & 1 \\
\hline $\begin{array}{l}\text { Mitra rufomaculata } \\
\text { Mitrella sp. }\end{array}$ & $\begin{array}{c}C \\
C\end{array}$ & sm & & & 1 & 5 \\
\hline 25. Morula granulata & $\stackrel{C}{C}$ & $\begin{array}{l}\text { phy, crs } \\
\text { crs }\end{array}$ & & 1 & & $\begin{array}{l}5 \\
1\end{array}$ \\
\hline 26. Morula uva & C & $\mathrm{mi}$ & & 4 & 1 & \\
\hline $\begin{array}{l}\text { Nassa serta } \\
\text { 27. Nassarius albescens }\end{array}$ & $\mathrm{N}, \mathrm{C}$ & $\mathrm{mi}$ & & & 1 & 1 \\
\hline gemmuliferus & $N$ & $\mathrm{sm}, \mathrm{crs}$ & & 16 & 16 & 14 \\
\hline 28. Nassarius arcularia & & & & & & \\
\hline $\begin{array}{l}\text { plicatus } \\
\text { Neritopsis radula }\end{array}$ & $\begin{array}{l}\mathrm{N} \\
\mathrm{C}\end{array}$ & $\begin{array}{l}\mathrm{sm} \\
\mathrm{m} \overline{\mathrm{i}}\end{array}$ & & 4 & 1 & 1 \\
\hline Planaxis lineolatus & $\mathrm{H}$ & phy, crs & $*$ & & & 11 \\
\hline Pleurotoma sp. & $\mathrm{C}$ & $\mathrm{sm}$ & & & 2 & \\
\hline Polinices mammilla & $\mathrm{C}$ & $\mathrm{sm}$ & & & & 1 \\
\hline 29. Polinices melanostoma & $\mathrm{C}$ & phy, sm & & 1 & & \\
\hline Rhinoclavis aspera & $\mathrm{D}$ & $\mathrm{sm}$ & & & & 1 \\
\hline Royella sinon & $\mathrm{D}, \mathrm{H}$ & crs & & & 1 & \\
\hline 30. Scala jomardi & $\mathrm{C}$ & phy & & 2 & 1 & \\
\hline Scala lamellosa & C & $\mathrm{mi}$ & 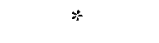 & & & 1 \\
\hline 31. Serpulorbis inopertus & $s \mathrm{M}$ & phy, crs & & 51 & 50 & 31 \\
\hline 32. Stomatia phymotis & $\mathrm{H}$ & phy & & 2 & & 1 \\
\hline 33. Strombus erytbrinus & $\mathrm{H}$ & phy & $*$ & 3 & & 1 \\
\hline Strombus fusiformis & $\mathrm{H}$ & crs & & & & 1 \\
\hline $\begin{array}{l}\text { 34. Strombus gibberulus albus } \\
\text { 35. Strombus mutabilis }\end{array}$ & $\mathrm{H}$ & phy & $*$ & 11 & 3 & \\
\hline $\begin{array}{l}\text { 50. Stromous mutalus } \\
\text { ocbroglottis }\end{array}$ & $\mathrm{H}$ & phy, crs & & 21 & 17 & 10 \\
\hline Strombus tricornis & $\mathrm{H}$ & $\mathrm{sm}$ & & & & 1 \\
\hline Subancilla annulata & $\mathrm{C}$ & $\mathrm{sm}$ & & & 1 & \\
\hline Subancilla flammea & $\mathrm{C}$ & $\mathrm{sm}$ & & & 1 & \\
\hline Terebra affinis & $\mathrm{C}$ & $\mathrm{sm}$ & & & & 1 \\
\hline 36. Terebra babylonia & $\mathrm{C}$ & $\mathrm{sm}$ & & 1 & & \\
\hline 37. Terebra crenulata & $\mathrm{C}$ & $\mathrm{sm}$ & & 2 & 5 & 1 \\
\hline Terebra flavofasciata & $\mathrm{C}$ & sm & & & & 1 \\
\hline Thais tuberosa & $\mathrm{C}$ & mi, crs & & & & 2 \\
\hline 38. Trivia oryza & $\mathrm{C}$ & crs & & 1 & & \\
\hline 39. Trocbus dentatus + & $\mathrm{H}$ & phy, crs & & 1 & & \\
\hline 40. Trochus sp. & $\mathrm{H}$ & phy, crs & 0 & 1 & & 1 \\
\hline 41. Turbo radiatus & $\mathrm{H}$ & phy & & 14 & 9 & 8 \\
\hline Turritella maculata & $\mathrm{D}$ & $\mathrm{sm}$ & & & & 1 \\
\hline Vexillum amabile & C & phy, crs & & & 2 & \\
\hline Vexillum infaustum & $\mathrm{C}$ & phy, sm & & & & 1 \\
\hline 42. Vexillum leucozonias & $\mathrm{C}$ & phy, crs & 0 & 4 & 7 & \\
\hline 43. Vexillum pardalis & $\mathrm{C}$ & crs & & 4 & 3 & \\
\hline Volema pyrum nodosum + & $\mathrm{N}, \mathrm{C}$ & $\mathrm{sm}$ & & & 1 & \\
\hline 44. Xenoturris cingulifera & $\mathrm{C}$ & $\mathrm{sm}$ & & 2 & 3 & \\
\hline 45. Dentalium elephantinum + & $\mathrm{D}$ & $\mathrm{sm}$ & & 1 & 1 & \\
\hline Dentalium cf. octangulum & $\mathrm{D}$ & $\operatorname{sm}$ & & & 1 & \\
\hline Arca antiquata & $\mathrm{sF}$ & $\mathrm{mi}, \mathrm{crs}$ & & & 1 & 1 \\
\hline Arca plicata & $\mathrm{sF}$ & $\mathrm{mi}$ & & & 2 & 4 \\
\hline 46. Asaphis deflorata & $\mathrm{mF}$ & $\mathrm{sm}$ & & 17 & & 3 \\
\hline 47. Barbatia lacerata & $s F$ & crs & & 2 & 1 & 3 \\
\hline Cardium australe & $\mathrm{mF}$ & $\mathrm{sm}$ & & & & 1 \\
\hline
\end{tabular}


Tabelle 6 (2. Fortsetzung)

\begin{tabular}{|c|c|c|c|c|c|c|}
\hline \multirow{2}{*}{$\begin{array}{l}\text { Species } \\
\text { Cardita variegata }\end{array}$} & \multirow{2}{*}{$\begin{array}{l}\text { Ernäh- } \\
\text { rungs- } \\
\text { weise" }\end{array}$} & $\begin{array}{l}\text { Bevorzugtes } \\
\text { Habitat bzzw. } \\
\text { Substrat }\end{array}$ & $\begin{array}{l}\text { Saisonale } \\
\text { Abwande- } \\
\text { rung in }\end{array}$ & \multicolumn{3}{|c|}{$\begin{array}{l}\text { Anz. d. Individuen währ. } \\
\text { d. Untersuchungsper. im } \\
\text { Febr. } 76 \text { Juni } 76 \text { Nov. } 76\end{array}$} \\
\hline & & $\mathrm{mi}$ & & & 1 & \\
\hline Chama sp. & $s \mathbf{F}$ & crs & & 1 & 4 & 4 \\
\hline Codackia divergens & $\mathrm{mF}$ & $\mathrm{sm}$ & & & 1 & 5 \\
\hline 48. Cyclotellina scobinata & $\mathrm{mF}$ & $\mathrm{sm}$ & & 5 & 1 & 1 \\
\hline Gastrochaena sp. & $\mathrm{sF}$ & $\mathrm{mi}, \mathrm{crs}$ & & & 2 & 2 \\
\hline Glycymeris pectunculus & $\mathrm{mF}$ & $\mathrm{sm}$ & & & 1 & \\
\hline 49. Hemicardium fragum & $\mathrm{mF}$ & phy, sm & & 33 & 19 & 14 \\
\hline Isognomon sp. & $s F$ & & & & & 1 \\
\hline Lima lima & $\mathrm{mF}$ & $\mathrm{sm}, \mathrm{crs}$ & & & & 1 \\
\hline Loripes erythraeus & $\mathrm{mF}$ & $\mathrm{sm}$ & & & & $i$ \\
\hline Lucina edentula & $\mathrm{mF}$ & $\mathrm{sm}$ & & 15 & 26 & 19 \\
\hline 50. Modiolus auriculatus & $s \mathrm{~F}$ & phy, sm, crs & & 31 & 18 & 12 \\
\hline 51. Notirus macrophyllus & $s F$ & mi, crs & & 1 & 4 & 7 \\
\hline 52. Pecten sp. + & $\pm \mathrm{mF}$ & $\mathrm{sm}$ & & 5 & 4 & 1 \\
\hline 53. Pedum spondyloideum & & $\mathrm{mi}$ & & 12 & 4 & 2 \\
\hline Pinctada radiata & $\mathrm{sF}$ & $\mathrm{mi}, \mathrm{crs}$ & & & & 1 \\
\hline 54. Pinna bicolor & $s F$ & $\mathrm{sm}$ & & 2 & 3 & 1 \\
\hline 55. Streptopinna saccata & $s \mathrm{~F}$ & $\mathrm{mi}$ & & 3 & 2 & 1 \\
\hline 56. Tapes litteratus & $\mathrm{mF}$ & $\mathrm{sm}$ & & 50 & 17 & 10 \\
\hline 57. Tridacna squamosa & $\mathrm{sF}$ & $\mathrm{mi}, \mathrm{crs}$ & & 7 & 5 & 4 \\
\hline \multicolumn{3}{|c|}{ Gesamtindividuenanzahl der Mollusca } & & 477 & 378 & 292 \\
\hline \multicolumn{3}{|c|}{ Prozentzahl bezogen auf Februar 1976} & & $100 \%$ & $79,2 \%$ & $61,2 \%$ \\
\hline
\end{tabular}

Bei den 76 Carnivoren und Nekrophagen war eine gewisse Bevorzugung algenfreier Hartsubstrate unverkennbar, bei einem Teil der räuberischen Gastropoden und bei den 125 mobilen Filtrierern dagegen eine solche von Weichböden. Alle 133 sessilen Filtrierer und Leimrutenfänger wurden auf Korallenfelsstrukturen gefunden, die meisten auf toten Flächen im Phytalbereich, andere aber wie Dendropoma maximum und Pedum spondyloideum inmitten lebenden Korallengewebes der Mikroatolle. Quantitativ belegbare Abhängigkeiten zwischen Ernährungweise und Habitatwahl lassen sich aus den oben gegebenen Zahlen freilich noch nicht ableiten.

Die Abnahme der Gesamtindividuenzahl im Testareal L-1 (Tab. 6) vom Februar 1976 (100\%) über Juni 1976 (79,2\%) bis November 1977 (61,2\%) gleichzeitig mit dem Rückgang der Makroalgenbestände im Frühsommer und ihrem völligen Verschwinden im Spätherbst legt einen Zusammenhang zwischen Abundanz und Diversität der Mollusken und der saisonalen Phytalregression nahe. Bei Aufgliederung der jeweiligen Molluskenpopulation nach ihren Ernährungsweisen zeigen die herbivoren und detritophagen Arten innerhalb von 16 Monaten eine weit stärkere Abundanzabnahme (Bestand im November 1977: 60,0\%) als die carnivoren und nekrophagen (79,7\%). Zur gleichen Zeit war der Rückgang bei den mikrophagen Arten noch größer (57,1 \%). Bemerkenswerte Veränderungen erfuhren auch die Individuenzahlen während der ersten Phase der Phytalregression zwischen Februar und Juni 1976: Die Anzahl der Herbivoren und Detritophagen ging auf $22,9 \%$ zurück, während die der Carnivoren und Nekrophagen um 8,1 \% zunahm. Weitere 
Aufschlüsse werden von den Mageninhaltsanalysen erwartet, die Mastaller (in Vorbereitung) an 15 Molluskenarten aus der Rifflagune vornahm. Insgesamt war die Individuenzahl bei den Filtrierern am höchsten und bei den räuberischen Arten am niedrigsten, während die Artenzahl bei den Carnivoren und Nekrophagen am höchsten war und bei den Filtrierern am niedrigsten. Auf jeden Fall aber sind allein mit den 258 Filtrierern und Leimrutenfängern mehr als die Hälfte aller 477 im Februar. 1976 gefundenen Molluskenindividuen vom Zustand des Phytals unabhängig. Immerhin wurde bei einigen Arten eine Tendenz zur Abwanderung in andere Riffzonen während der Phytalregression im Frühsommer beobachtet. Sie war teils in die ufernähere "immergrüne" Halodule uninervis Zone gerichtet (10 Arten), teils in die seewärts liegende Mikroatollzone (5 Arten).

\section{Echinodermata}

Im Gegensatz zu anderen mobilen Tiergruppen ließen sich bei den meisten Echinodermen quantitative Nachweise ihrer saisonalen Bestandsänderungen ohne ernsthafte Eingriffe in das Gefüge ihres Biotopes führen: So verändert Halodeima ihren Standort auf Sandmulmflächen und im Phytal oft tagelang nur geringfügig und gerade die in der Rifflagune häufigsten Seeigelarten wie Echinometra mathaei und Diadema setosum sind während des Tages praktisch immer auf die gleichen Schlupfwinkel beschränkt, letztere vor allem auf die basalen Höhlungen der beiden Mikroatolle (Abb. 8). Der Seestern Linckia multiflora ist dagegen häufig auch während der hellsten Tagesstunden offen im Habitat zu finden. Mit Ausnahme der nicht vollständig erfaßbaren Schlangensterne müssen also die Abundanzangaben in Tabelle 7 als komplett gelten. Dabei können die übrigen, im Testareal L-1 nur in Einzelexemplaren gefundenen Echinodermen bei den nachstehenden Überlegungen unberücksichtigt bleiben.

Im Oktober 1975 wurde ein Gesamtbestand von 79 durchwegs adulten Individuen aus 7 Arten festgestellt. Unter ihnen waren 49 Echinometra mathaei, 9 Diadema setosum, 8 Linckia multiflora, 7 Ophiocoma spp. und 6 Halodeima spp. Im Februar 1976 wurde bei allen diesen Arten eine bemerkenswerte Individuenzunahme beobachtet, so bei Echinometra auf 129 Tiere, bei Diadema auf 17, bei Linckia auf 16, bei Ophiocoma auf 17 und bei Halodeima auf 9. Auffallender als die Vergrößerung des Gesamtbestandes auf 196 Individuen aus 13 Arten war dabei der nunmehr hohe Anteil juveniler Tiere mit etwa einem Drittel (62), unter denen sich 43 Echinometra, 7 Diadema, 6 Linckia und 4 Halodeima befanden. Etwa $77 \%$ der adulten Individuen und fast $85 \%$ der juvenilen gehörten herbivoren Seeigelarten an. Ein enger Zusammenhang zwischen Zuwanderung dieser Echinodermen und Aufkommen des Algenwuchses mit Beginn des Jahres 1976 darf daher angenommen werden. Damit verbundene Beobachtungen werden später noch diskutiert (vgl. p. 504).

\section{Pisces}

Ohne detaillierte Beobachtungen zur Abundanz der Fischfauna vorzunehmen, wurden doch sämtliche Dauerbewohner, Gelegenheitsbesucher und Irrgäste des Testareals L-1 registriert (Tab. 8): Unter den 14 erkannten Arten befanden sich, vermutlich aus Mangel an geeigneten Verstecken, nur wenige Dauerbewohner mit eigenem Revier. Zu ihnen können eigentlich nur ein Hemibalistes chrysopterus gerechnet werden, der eine kleine Felsboden- 
höhle an der NO-Ecke des Areals bewohnte, und ein Ampbiprion bicinctus in einer Radianthus koseirensis (Actiniaria) unmittelbar außerhalb der Westgrenze des Areals, der dieses aber teilweise als Revier verteidigte. Auch ein adulter Dascyllus trimaculatus wurde schon im Oktober 1975 unter dem großen Goniastrea retiformis - Microatoll beobachtet und dort auch noch im Februar 1976 vorgefunden, während ein Jungtier erst seit Februar den schmalen Tunnel des benachbarten Cyphastrea microphthalma-Mikroatolls bezogen hatte. Uber einen längeren Zeitraum hinweg wurde je ein Individuum von Sidera grisea, Dendrochirus brachypterus und Meiacanthus nigrolineatus im Areal angetroffen. Alle anderen identifizierten Arten müssen entweder als öfters auftauchende Gelegenheitsbesucher bezeichnet werden wie ein Chaetodon chrysurus paucifasciatus, ein Pterois volitans

Tabelle 7

Ernährungsweise, Habitatpräferenz und saisonale Abundanz der Echinodermata im Testareal L-1. Die Arten Nr. 1-13 wurden am 26. und 29. Oktober 1975 sowie am 7. und 8. Februar 1976 kartiert und in Abb. 8 bei senkrechter Projektion lagerichtig eingetragen. Von den anderen Arten wurden bis November 1977 nur Belegexemplare ohne Fundortangaben gesammelt. * - Kein typischer Rifflagunenbewohner. $* *$ - Kein typischer Flachwasserbewohner. *Abkürzungen in Tabelle 6. *** Abkürzungen in Tabelle 2

\begin{tabular}{|c|c|c|c|c|c|c|}
\hline \multirow{3}{*}{ Species } & \multirow{3}{*}{$\begin{array}{l}\text { Ernäh- } \\
\text { rungs- } \\
\text { weise }^{*}\end{array}$} & \multirow{3}{*}{$\begin{array}{l}\text { Bevorzugtes } \\
\text { Habitat bzw. } \\
\text { Substrat }^{2 * *}\end{array}$} & \multicolumn{4}{|c|}{ Saisonale Abundanz im } \\
\hline & & & Oktobe & I 1975 & Februar & 1976 \\
\hline & & & $\begin{array}{c}\text { Anzahl } \\
\text { der } \\
\text { Individuen }\end{array}$ & $\begin{array}{c}\text { dar- } \\
\text { unter } \\
\text { juvenile }\end{array}$ & $\begin{array}{c}\text { Anzahl } \\
\text { der } \\
\text { Individuen }\end{array}$ & $\begin{array}{c}\text { dar- } \\
\text { unter } \\
\text { juvenile }\end{array}$ \\
\hline 1. Halodeima atra & $\mathrm{D}$ & phy, sm, crs & 3 & 0 & 5 & 3 \\
\hline 2. Halodeima edulis & $\mathrm{D}$ & phy, sm, crs & 3 & 0 & 4 & 1 \\
\hline Holotburia pardalis & $\mathrm{D}$ & $\mathrm{sm}$ & & & & \\
\hline Pseudocnus nov. spec. & $\mathrm{D}$ & crs & & & & \\
\hline 3. Clypeaster bumilis & $\mathrm{D}$ & $\mathrm{sm}$ & 0 & 0 & 1 & 0 \\
\hline 4. Diadema setosum & $\mathrm{H}$ & phy, mi, crs & 9 & 0 & 17 & 7 \\
\hline 5. Echinometra mathaei & $\mathrm{H}$ & phy, mi, crs & 49 & 0 & 129 & 43 \\
\hline $\begin{array}{l}\text { 6. Eucidaris metularia } \\
\text { 7. Heterocentrotus }\end{array}$ & $\mathrm{H}$ & $\mathrm{mi}$, crs & 0 & 0 & 1 & 1 \\
\hline mammillatus: & $\mathrm{H}$ & crs & 0 & 0 & 1 & 1 \\
\hline Lovenia elongata & $\mathrm{D}$ & $\mathrm{sm}$ & & & & \\
\hline Metalis spatagus & $\mathrm{D}$ & $\mathrm{sm}$ & & & & \\
\hline 8. Microcyphus rousseaui: & $\mathrm{H}$ & crs & 0 & 0 & 1 & 0 \\
\hline Paraster gibberulus & $\mathrm{D}$ & $\mathrm{sm}$ & & & & \\
\hline 9. Tripneustes gratilla & $\mathrm{H}$ & phy, mi, crs & 0 & 0 & 3 & 0 \\
\hline $\begin{array}{l}\text { 10. Asterina burtoni } \\
\text { Astropecten bemprichi }\end{array}$ & $\begin{array}{l}\mathrm{N}, \mathrm{C} \\
\mathrm{C}\end{array}$ & $\begin{array}{l}\mathrm{mi}, \mathrm{crs} \\
\mathrm{sm}\end{array}$ & 0 & 0 & 1 & 0 \\
\hline 11. Linckia multiflora & $\mathrm{N}, \mathrm{C}$ & $\mathrm{sm}, \mathrm{mi}, \mathrm{crs}$ & 8 & 0 & 16 & 6 \\
\hline Amphipholis squamata & $\mathrm{D}$ & crs & & & & \\
\hline Dougaloplus echinatus & $\mathrm{D}$ & crs & & & & \\
\hline 12. Opbiocoma erinaceus & $\mathrm{D}$ & $\mathrm{mi}, \mathrm{crs}$ & 2 & 0 & 5 & 0 \\
\hline Ophiocoma pica & $\mathrm{D}$ & $\mathrm{mi}$ & & & & \\
\hline 13. Opbiocoma scolopendrina & $\bar{F}, \mathrm{D}$ & $\mathrm{mi}$, crs & 5 & 0 & 12 & 0 \\
\hline Ophiolepis cincta & $\mathrm{D}$ & $\mathrm{mi}$, crs & & & & \\
\hline Opbionereis dubia & $\mathrm{D}$ & $\mathrm{mi}$ & & & & \\
\hline Ophionereis porrecta & F, D & $\mathrm{mi}, \mathrm{crs}$ & & & & \\
\hline Opbiotbrix propinqua & F, D & $\mathrm{mi}, \mathrm{crs}$ & & & & \\
\hline \multirow{2}{*}{\multicolumn{3}{|c|}{ Gesamtindividuenanzahl der Echinodermata }} & & & & \\
\hline & & & 79 & 0 & 196 & 62 \\
\hline
\end{tabular}


und ein Synodus variegatus, oder als typische, nur einmal beobachtete Irrgäste. Zu ihnen zählten Bothus pantherinus, Paracirrbites forsteri, Platycephalus indicus, Pteropterus radiatus und Synanceichthys verrucosa.

\section{DISKUSSION}

Daß Korallenriffe zu den dichtest und vielfältigst bevölkerten Lebensräumen gehören, ist schon seit langem bekannt, ohne daß hierfür quantitative Nachweise vorliegen: Nur von wenigen Riffgebieten bestehen umfassende Artenlisten aller vorgefundenen Tiergruppen und noch seltener sind sie mit brauchbaren ökologischen Hinweisen und gesicherten Abundanzangaben versehen. So haben Mergner \& Schuhmacher (1974) für zwei Saumriffabschnitte bei Aqaba je etwa 200 Arten erfaßt und Angaben der relativen Häufigkeit dieser Arten in den einzelnen Riffzonen veröffentlicht. Nunmehr wird zum erstenmal wenigstens für einen räumlich begrenzten Riffabschnitt und zumindest für ein volles Winterhalbjahr eine quantitative Analyse der Abundanz, Dominanz und Diversität aller MakrobenthosGruppen sowie ihrer saisonalen Veränderungen gegeben.

Viele geomorphologisch begründbare Anzeichen deuten auf ein hohes Alter des Lagunensaumriffes südlich von Aqaba hin und darauf, daß seine Lagune das Ergebnis eines langfristigen Erosionsprozesses im Bereich des früheren Riffdaches darstellt (Mergner \& Schuhmacher, 1974). Dafür spricht auch der nahezu stabile Zustand der Lagunenbesiedlung und der sie tragenden Lebensgemeinschaften. Er läßt sich zweifellos als KlimaxStadium beschreiben, wie es vor allem auch in der Ausgeglichenheit von saisonalem Schwund und Neuzuwachs bei mehreren sessilen Makrobenthosgruppen wie Algen und

Tabelle 8

Habitatpräferenz und Abundanz der Pisces im Testareal L-1 während der Untersuchungsperioden im Oktober 1975 und Februar/März 1976. *-Ständige Bewohner des Testareals. **-Gelegenheitsbesucher und Irrgäste. * Abkürzungen in Tabelle 2

\begin{tabular}{|c|c|c|c|}
\hline Species & $\begin{array}{l}\text { Bevorzugtes } \\
\text { Habitat bzw. } \\
\text { Substrat" }\end{array}$ & \multicolumn{2}{|c|}{$\begin{array}{l}\text { Anz. d. Individuen währ. } \\
\text { d. Untersuchungsper. im } \\
\text { Oktober } 75 \quad \text { Februar } 76\end{array}$} \\
\hline Synodus variegatus & $\mathrm{mi}, \mathrm{crs}$ & & 1 \\
\hline Siderea griseat & $\mathrm{mi}, \mathrm{crs}$ & 1 & 1 \\
\hline Bothus pantherinus** & phy, sm & 1 & \\
\hline Amphiprion bicinctus" & Radianthus koseirensis & 1 & 1 \\
\hline Chaetodon chrysurus paucifasciatus: & $\mathrm{mi}, \mathrm{crs}$ & 1 & 1 \\
\hline Dascyllus trimaculatus" & $\mathrm{mi}$ & 1 & 2 \\
\hline Dendrochirus brachypterus* & $\mathrm{mi}, \mathrm{crs}$ & & 1 \\
\hline Meiacantbus nigrolineatus: & $\mathrm{mi}$ & 1 & 1 \\
\hline Paracirrbites forster $* *$ & $\mathrm{mi}, \mathrm{crs}$ & 1 & \\
\hline Platycepbalus indicustre & $\mathrm{sm}$ & 1 & \\
\hline Pterois volitans: & $\mathrm{mi}$ & & 1 \\
\hline Pteropterus radiatus & $\mathrm{mi}$ & & 1 \\
\hline Synanceichtbys verrucosa $a^{\text {*3: }}$ & crs & & 1 \\
\hline Hemibalistes chrysopterus" & crs & 1 & 1 \\
\hline \multicolumn{2}{|c|}{ Gesamtindividuenanzahl der Pisces $(*-6 ; * *-9)$} & 9 & 12 \\
\hline
\end{tabular}


Cnidariern (so bei Stylopbora pistillata, Sinularia polydactyla und Xenia membranacea) zum Ausdruck kommt. Daß nach Loya (1976) der jährliche Zuwachs junger StylopboraKolonien mit nur 1,9-2,5 cm geringer ist als nach den eigenen Messungen, spielt bei diesen Feststellungen nur eine untergeordnete Rolle. Da sich nämlich die meisten der 65 jungen Kolonien in unmittelbarer Nähe der wenigen großen Kolonien angesiedelt haben, ist zumindest nicht auszuschließen, daß sie aus deren Bruttätigkeit stammen. Auch die Beobachtung, daß im Bereich des Testareals L-1 bei mehreren Tiergruppen wie den Mollusken und Echinodermen in andere Riffzonen gerichtete Wandertendenzen zu lokalen Abundanzschwankungen führen, steht der Annahme eines Klimax-Stadiums nicht entgegen. Freilich könnten nur langwierige autökologische Untersuchungen an einer Vielzahl markierter Individuen klären, ob es sich dabei um endogen verursachte, dauernde Populationsänderungen handelt oder nur um jährlich wiederkehrende, gerichtete Wanderbewegungen in unmittelbarem Zusammenhang mit den saisonalen Bestandsschwankungen der benthischen Makroalgen.

Die besondere Rolle der Echiniden beim Verschwinden der Algenpopulationen geht aus zahlreichen eindeutigen Beobachtungen hervor. Sie belegen u. a. die Zuwanderung herbivorer Seeigel mit Aufkommen des Algenwuchses, registrieren dabei die starke Zunahme juveniler Individuen und erklären das Vorkommen der auffälligen, nahezu algenfreien Areale im Umkreis der Diadema setosum-Verstecke in den basalen Höhlungen der beiden Mikroatolle: Tatsächlich wurden von den 17 Diadema-Individuen des Testareals allein 15 unter den beiden Mikroatollen festgestellt und die beiden restlichen unter großen Korallenhorsten (Abb. 8). Durch Käfigexperimente konnte Mastaller (in Vorbereitung) die Weidetätigkeit von Seeigeln als Ursache für das Entstehen derartiger „Halos“ nachweisen. Da im gleichen Areal mit etwa $1 \mathrm{~m}$ Durchmesser im Februar 1976 auch sämtliche Xenia-Kolonien verschwunden waren und 18 von ihnen sogar näher als $0,5 \mathrm{~m}$ von den genannten Diadema-Schlupfwinkeln entfernt lagen, muß Weidefraß von Seeigeln während der algenarmen Periode vom Spätherbst bis zum Jahresbeginn auch hierfür verantwortlich gemacht werden (vergleiche Abb. 3 und 8; siehe auch Mergner \& Svoboda, 1977). Natürlich kann bei den beiden mehrjährig ausdauernden Xenia-Arten auch ein Zusammenbruch durch Erschöpfung am Ende der langen sommerlichen Brutsaison angenommen werden (Gohar, 1940). Auch Vine (1974) und Benayahu \& Loya (1977) weisen auf den bedeutenden Einfluß hin, den einerseits territoriale Korallenbarsche wie Pomacentrus lividus, anderseits weidende Seeigel auf die Algenentwicklung nehmen. Offensichtlich üben also herbivore Echiniden wie Diadema setosum und Tripneustes gratilla eine wichtige Kontrollfunktion bei der Bestandsentwicklung der benthischen Makroalgen und Weichkorallen aus. Außerdem beeinflussen sie durch Detritusbildung auch die Ansiedlung anderer sessiler Rifforganismen in unmittelbarer Nachbarschaft ihrer Schlupfwinkel (Schuhmacher, 1974).

Nach Benayahu \& Loya (1977) und Mastaller (in Vorbereitung) könnte der auffällige Zuwachs herbivorer Seeigelpopulationen ein Indikator für tiefgreifende Störungen des ökologischen Gleichgewichtes im nördlichen Golf von Aqabạ sein: Vor allem die Massenvermehrung von Tripneustes gratilla, Echinothrix diadema und Diadema setosum deutet auf eine ernsthafte Gefährdung des bislang ausgewogenen Zustandes in der Artenzusammensetzung und Besiedlungsdichte ursprünglich vielgestaltiger benthischer Lebensgemeinschaften der Flachwasserregion hin. Zunehmende Ölverschmutzung und Eutrophierung 
Quantitative ökologische Analyse

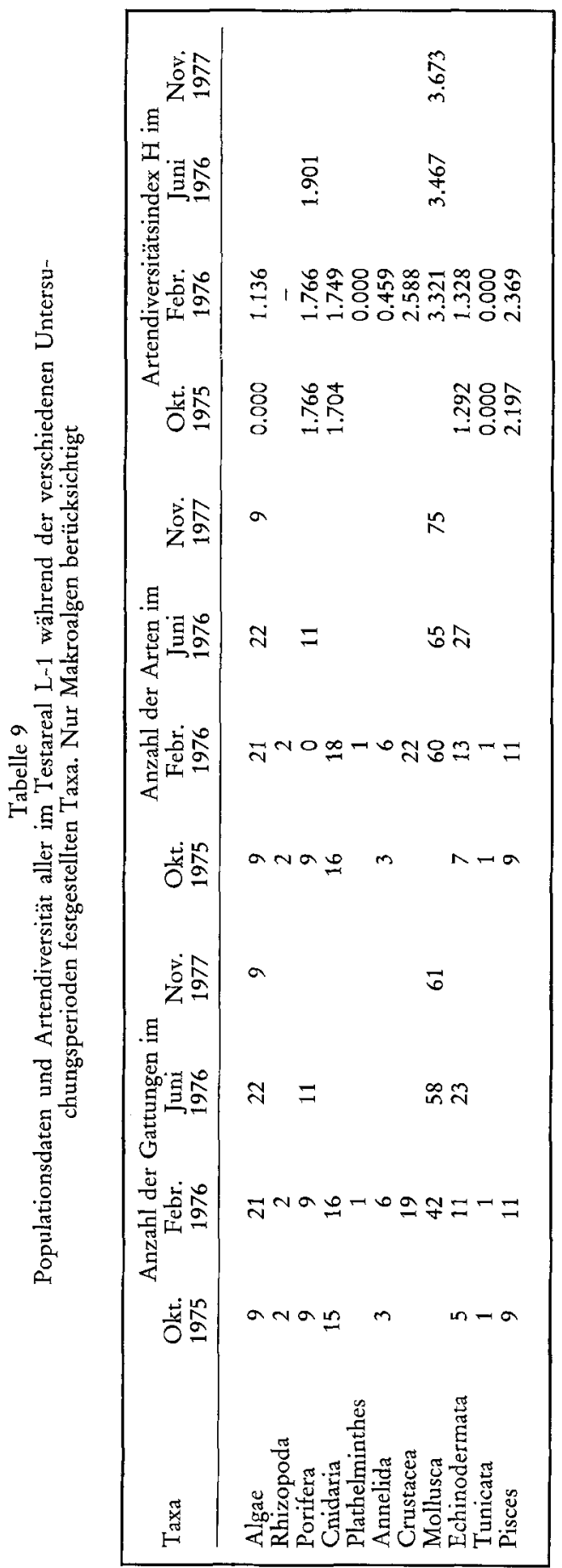


durch Phosphatstaubeinwehung, Abwässereinleitung und andere menschliche Einflüsse können also an den Biozönosen innerhalb relativ kurzer Zeit irreversible Veränderungen bewirken, welche die natürlichen Folgen des langfristigen erosionsbedingten Riffabbaues bei weitem übertreffen. Daher sind, auf längere Sicht gesehen, auch im Golf von Aqaba ähnliche nachteilige Folgen zu befürchten, wie sie schon seit vielen Jahren an bestimmten Mittelmeerküsten beobachtet werden.

Während der Eingangsuntersuchungen im Oktober 1975 konnten aus Gründen der Schonung des Biotopes und seiner Lebensgemeinschaften von bestimmten Tiergruppen (Foraminiferen, Serpuliden, Crustaceen, Gastropoden und Ophiuriden) nur Belegproben entnommen werden. Bei den übrigen Tiergruppen und den Makroalgen war dagegen von Anbeginn eine quantitative Erfassung aller Individuen bzw. Kolonien möglich. Die Abundanzangaben sind daher unterschiedlich zu bewerten. Doch erlauben die ganzjährigen Kontrollen bei den Mollusken und einem großen Teil der Echinodermen sowie die komplette Halbjahresanalyse beim sessilen Makrobenthos einen guten Uberblick über die jahreszeitlichen Veränderungen und die verschiedenen Diversitätsmuster im Testareal (Tab. 9).

Insgesamt wurden im Februar 1976 einschließlich der später noch aufgesammelten Mollusken mehr als 2000 Algenkolonien und 2200 Individuen bzw. Kolonien der Makrofauna erfaßt. Mit 237 Arten und 192 Gattungen aus 4 Algenstämmen und 10 Tierstämmen auf nur $25 \mathrm{~m}^{2}$ einer wenig abwechslungsreichen Lagunenlandschaft übertreffen diese Abundanzdaten bei weitem die früher erzielten Sammelergebnisse aus den fast $6000 \mathrm{~m}^{2}$ großen Riffabschnitten südlich von Aqaba (Mergner \& Schuhmacher, 1974). Außerdem vermitteln sie für die meisten Gruppen ein fast vollständiges Bild ihrer Besiedlungsstruktur und ermöglichen eine umfassende quantitative Analyse der im Testareal angetroffenen Biozönosen. Dabei ist der Widerspruch zwischen ihrer hohen Besiedlungsdichte und dem doch schwach strukturierten Siedlungsraum nur scheinbar. Das Zusammentreffen verschiedener biophysiographischer Riffzonen ergibt eine Vielzahl unterschiedlicher Mikrohabitate, die wiederum ökologische Nischen darstellen für spezifische Lebensgemeinschaften mit entsprechenden Anpassungsstrategien. So bietet der zunächst so einförmig erscheinende Lagunenbiotop L-1 offensichtlich ausreichende Versteckmöglichkeiten im Korallenfels und anderen geeigneten Substraten z. B. für eine artenreiche, aber unterschiedlich spezialisierte Molluskenfauna: 59,1\% aller Arten lebten kryptisch an Hartsubstrat gebunden, während 31,4 \% typische Sandbewohner und 9,5\% Phytalbewohner waren. Daher erscheint es begründet, eine Kombination der größten Artenanzahl und des höchsten Artendiversitätsindex unter den mobilen Gruppen zu suchen (Tab. 9; siehe auch Mergner 8 Mastaller, 1979 im Druck).

Tatsächlich rührt der hohe Diversitätsindex etwa bei den Crustaceen und Mollusken teilweise daher, daß in diesem sehr heterogenen Biotop viele Arten mit ähnlichen ökologischen Ansprüchen zusammenleben. Die vielfältigen Umweltbedingungen führen zu ebensolchen Varietäten an Raum- und Nahrungskonkurrenzen, woraus wiederum eine Zunahme an hochspezialisierten Arten resultiert. Bei den Crustaceen und Fischen könnte der hohe Diversitätsindex auch auf die große Artenzahl bei jeweils nur geringer Individuenzahl zurückzuführen sein. Dagegen weist ein niedriger Diversitätsindex auf die Dominanz einer oder weniger Arten hin, wie dies vorwiegend bei den sessilen Benthosgruppen, etwa bei den Makroalgen, der Fall ist. Die besonderen Probleme der Artendiver- 
sität und deren populationsbiologische Zusammenhänge bei Mollusken und Echinodermen wird Mastaller (in Vorbereitung) noch ausführlich erläutern.

Abschließend sollen weitere Untersuchungen ausgewählter Riffareale mit Langzeitbeobachtungen des gesamten Benthos angeregt werden, um Veränderungen in der Populationsdynamik ihrer Einzelgruppen, vor allem infolge von Störungen des ökologischen Gleichgewichts, durch Vergleich mit intakten Riffarealen richtig einschätzen zu können.

Danksagungen. Die Deutsche Forschungsgemeinschaft ermöglichte in dankenswerter Weise diese Untersuchungen. Besonderer Dank gebührt den Herren cand. rer. nat. M. Mastaller für sorgfältige Aufsammlung und Kartierung der Crustaceen und Mollusken sowie für Berechnung der Artendiversität, und F. Nobbe für Aufsammlung und Kartierung der Poriferen. Die Bestimmung des Belegmaterials übernahmen ganz oder teilweise: Prof. Dr. R. Schnetter (Algae), cand. rer. nat. F. Nobbe (Porifera), Dr. J. Versefeldt (Alcyonaria), Dr. H. Schuhmacher (Scleractinia), Dr. B. Ehlers (Turbellaria), Dr. G. Hartmann-Schröder und Dr. P. Knight-Jones (Polychaeta), Dr. L. B. Holthuis (Crustacea), cand. rer. nat. M. Mastaller (Mollusca, Echinodermata) und Dr. G. Paterson (Ascidiacea). Allen Genannten sei hier nochmals herzlich gedankt, ebenso Herrn F. Theissen für die sorgfältige Ausführung der Zeichnungsvorlagen sowie der Faculty of Science der University of Jordan für ihre Gastfreundschaft an der Marine Science Station Aqaba. Die Farbdrucke wurden aus Titel 52394, RUB, finanziert.

\section{ZITIERTE LITERATUR}

Benayahu, H. \& Loya, Y., 1977. Seasonal occurrence of benthic algae communities and grazing regulation by sea urchins at the coral reefs of Eilat, Red Sea. Proc. int. Coral Reef Symp., Miami 3 (1), 383-389.

Gohar, H. A. F., 1940. Studies on the Xeniidae of the Red Sea. Publs mar. biol. Stn Ghardaqa 2, 25-118.

Loya, Y., 1976. The Red Sea coral Stylophora pistillata is an $\mathrm{r}$ strategist. Nature, Lond. 259, 478-479.

Mastaller, M., 1978. The marine molluscan assemblages of Port Sudan, Red Sea. Zoöl. Meded., Leiden 53, 117-144.

- Beiträge zur Faunistik und Ókologie der Korallenriffe bei Aqaba, Rotes Meer, unter besonderer Berücksichtigung der Mollusken und Echinodermen. Diss., Univ. Bochum. (In Vorb.)

Mergner, H. \& Mastaller, M., 1979. Ecology of a reef lagoon area near Aqaba (Red Sea). Symp. coast. mar. Environm. Red Sea, Gulf of Aden and Trop. Western Indian Ocean Khartoum, 40 pp. (In press)

— \& Schuhmacher, H., 1974. Morphologie, Okologie und Zonierung von Korallenriffen bei Aqqaba (Golf von Aqaba, Rotes Meer). Helgoländer wiss. Meeresunters. 26, 238-358.

- Quantitative ökologische Analyse eines Vorriffareals bei Aqaba (Golf von Aqaba, Rotes Meer). (In Vorb.)

— \& Svoboda, A., 1977. Productivity and seasonal changes in selected reef areas in the Gulf of Aqaba (Red Sea). Helgoländer wiss. Meeresunters. 30, 383-399.

Nobbe, F. Beiträge zur Faunistik und Ókologie der Korallenriffe bei Aqaba, Rotes Meer, unter besonderer Berücksichtigung der Poriferen. Diss., Univ. Bochum. (In Vorb.)

Pielou, E. C., 1969. An introduction to mathematical ecology. Wiley-Interscience, New York, 286 pp.

Schuhmacher, H., 1974. On the conditions accompanying the first settlement of corals on artificial reefs with special reference to the influence of grazing sea urchins (Eilat, Red Sea). Proc. int. Coral Reef Symp., Brisbane 2 (1), 257-267.

Svoboda, A., 1978. In situ monitoring of oxygen production and respiration in Cnidaria with and without Zooxanthellae. In: Physiology and behaviour of marine organisms. Ed. by D. S. McLusky \& A. J. Berry. Pergamon Press, Oxford, 75-82.

Vine, P. J., 1974. Effects of algal grazing and aggressive behaviour of the Pomacentrus lividus and Acanthurus sohal on coral reef ecology. Mar. Biol. 24, 131-136. 